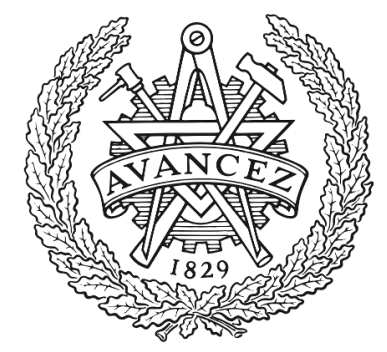

CHALMERS

UNIVERSITY OF TECHNOLOGY

\title{
Synthesis of cis-Oriented Vicinal Diphenylethylenes through a Lewis Acid-Promoted Annulation of Oxotriphenylhexanoates
}

Downloaded from: https://research.chalmers.se, 2023-04-26 06:56 UTC

Citation for the original published paper (version of record):

Kamlar, M., Henriksson, E., Cisarova, I. et al (2021). Synthesis of cis-Oriented Vicinal

Diphenylethylenes through a Lewis Acid-Promoted Annulation of

Oxotriphenylhexanoates. Journal of Organic Chemistry, 86(13): 8660-8671.

http://dx.doi.org/10.1021/acs.joc.1c00445

N.B. When citing this work, cite the original published paper. 


\title{
Synthesis of cis-Oriented Vicinal Diphenylethylenes through a Lewis Acid-Promoted Annulation of Oxotriphenylhexanoates
}

\author{
Martin Kamlar, Elin Henriksson, Ivana Císarová, Marcus Malo, and Henrik Sundén*
}

Cite This: J. Org. Chem. 2021, 86, 8660-8671

Read Online

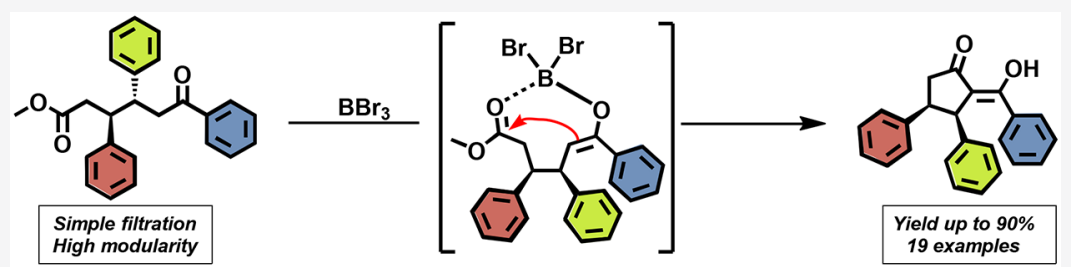

ABSTRACT: This study explores the synthesis of cyclic cis-vicinal phenyl ethylenes from oxotriphenylhexanoates. The reaction is a $\mathrm{BBr}_{3}$-promoted cyclization of 1,6-ketoesters (1) to five-membered diketo compounds (2). The synthesis is interesting as it constitutes one of the few examples of modular stereoselective synthesis of structures with a cis-oriented vicinal diphenylethylene. The core structure of $\mathbf{2}$ can be smoothly derivatized, which makes it a promising synthetic building block for further stereoselective synthetic applications.

\section{INTRODUCTION}

Cyclic compounds possessing adjacent $\mathrm{C}\left(\mathrm{sp}^{3}\right)$-aryl moieties are widely present in nature. While in most cases these compounds have a trans-vic-diphenylethylene moiety such as indanone $\mathrm{e}^{1-7}$ and reservatrol-based natural products, ${ }^{8-19}$ the cis-vic-diphenylethylene motif has been explored less. Nevertheless, these interesting substances still show biological activity, and representative examples include both pharmaceuticals $^{20}$ and natural products (Figure 1a). ${ }^{21-34}$ For example, the rocaglates, rocaglamide, and silvestrol have received widespread attention as they have been investigated for

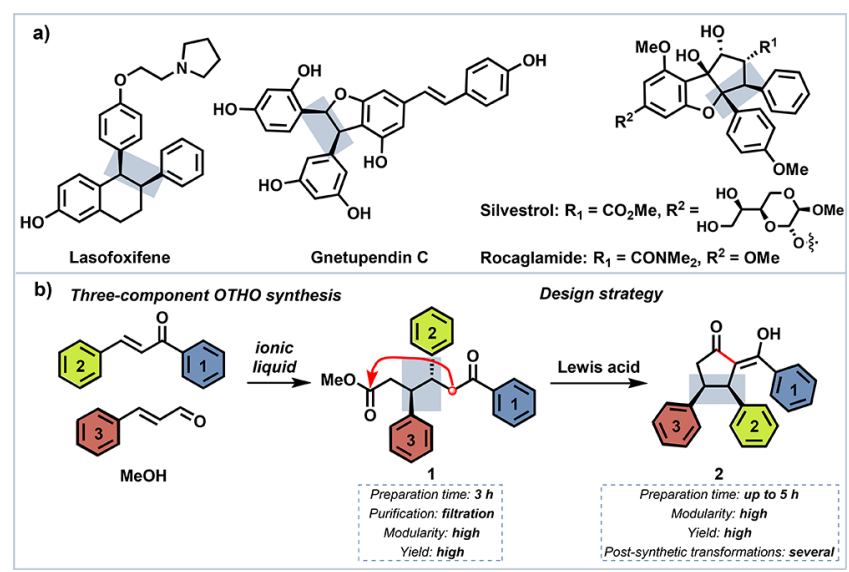

Figure 1. (a) Examples of biologically active compounds with a cisoriented diphenylethylene moiety. (b) Design approach for the synthesis of the desired carbocycle. therapeutic targets such as cancer ${ }^{21,34,35}$ and recently viruses like the coronavirus, ${ }^{36}$ the Ebola virus, ${ }^{37}$ and the hepatitis E virus. ${ }^{38}$ Thus, synthetic strategies leading to the formation the cis-vic-diphenylethylene are attractive. However, there are only a limited number of general methods for direct stereoselective formation of cyclic compounds possessing the cis-vic-diphenylethylene moiety. It is also worth noting that a majority of these syntheses are devoted to the diphenyl dihydrobenzofuran moiety found in the rocaglates. ${ }^{21,23,24,34,39-51}$

A practical way to prepare the cis-vic-configured $\mathrm{C}\left(\mathrm{sp}^{3}\right)$-aryl could potentially be a ring closure of an acyclic starting material with a preset configuration on the carbons bearing the aryl substituents. We envisioned that a Dieckmann type condensation of a 1,6-ketoester such as the oxotriphenylhexanoate $(\mathrm{OTHO})^{52-56}$ would be ideal for this type of transformation. The OTHO has an enolizable ketone and an ester moiety and can, after cyclization, generate a fivemembered diketo compound. Furthermore, one very interesting feature of the OTHO is that the synthesis of the OTHO proceeds with exclusive formation of the trans-vic-diphenylethylene. Used as a starting material in the Dieckmann condensation would thus ensure total control of the cis-vicdiphenylethylene buildup (Figure 1b).

Received: February 22, 2021

Published: June 17, 2021 


\section{RESULTS AND DISCUSSION}

Our studies commenced with screening of different Lewis acids for the cyclization of 1a (Table 1) because Lewis acids were

Table 1. Conditions for the Intramolecular Cyclization of $1 a^{a}$

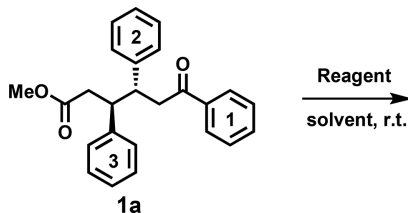

\begin{tabular}{|c|c|c|c|c|c|}
\hline entry & reagent & solvent & $\begin{array}{l}\text { time } \\
(\mathrm{h})\end{array}$ & $\begin{array}{c}\text { conversion } \\
(\%)\end{array}$ & $\begin{array}{c}\text { yield of } \\
\mathbf{2 a} / \mathbf{3} \\
(\%)^{b}\end{array}$ \\
\hline 1 & $\mathrm{BF}_{3} \cdot \mathrm{OEt}_{2}(1.0$ equiv $)$ & DCM & 168 & 0 & $0 / 0$ \\
\hline 2 & $\mathrm{BBr}_{3}$ (1.0 equiv) & DCM & 24 & 100 & $67 / 0$ \\
\hline 3 & $\mathrm{BCl}_{3}$ (1.0 equiv) & DCM & 48 & 100 & $60 / 0$ \\
\hline 4 & $\mathrm{AgOAc}$ (1.0 equiv) & DCM & 168 & 0 & - \\
\hline 5 & $\mathrm{SnCl}_{4}$ (1.0 equiv) & DCM & 168 & 0 & - \\
\hline 6 & $\mathrm{TiCl}_{4}$ (1.0 equiv) & DCM & 168 & 0 & - \\
\hline 7 & $\mathrm{AlCl}_{3}$ (4.0 equiv) & DCM & 168 & 0 & - \\
\hline 8 & $\mathrm{FeCl}_{3}$ (4.0 equiv) & DCM & 168 & 0 & - \\
\hline 9 & $\left(\mathrm{C}_{6} \mathrm{~F}_{5}\right)_{3} \mathrm{~B}$ (1.0 equiv) & DCM & 168 & 0 & - \\
\hline 10 & $\mathrm{NaH}$ (1.5 equiv) & THF & 6 & 50 & 45 \\
\hline 11 & $\mathrm{BBr}_{3}$ (1.5 equiv) & DCM & 3 & 100 & $80 / 0$ \\
\hline 12 & $\mathrm{BBr}_{3}$ (2.0 equiv) & DCM & 5 & 100 & $30 / 21$ \\
\hline 13 & $\mathrm{BBr}_{3}$ (3.0 equiv) & DCM & 5 & 100 & $11 / 28$ \\
\hline 14 & $\mathrm{BBr}_{3}(0.25$ equiv $)$ & DCM & 168 & 25 & $15 / 0$ \\
\hline 15 & $\mathrm{BBr}_{3}$ (1.5 equiv) & $\mathrm{CHCl}_{3}$ & 20 & 100 & $50 / 0$ \\
\hline 16 & $\mathrm{BBr}_{3}$ (1.5 equiv) & toluene & 16 & 100 & $63 / 0$ \\
\hline
\end{tabular}

${ }^{a}$ Reactions were carried out on a $0.25 \mathrm{mmol}$ scale of 1a in DCM (3 $\mathrm{mL}$ ) in a sealed reaction vessel at room temperature. ${ }^{b}$ Isolated yield.

already reported to be useful promoters in the Dieckmann type cyclization. ${ }^{57}$ Initial attempts showed that the cyclization reaction was highly dependent on the Lewis acid used. Only $\mathrm{BBr}_{3}$ and $\mathrm{BCl}_{3}$ delivered the corresponding cyclized product $2 \mathrm{a}$ in good yield after 24 and $48 \mathrm{~h}$, respectively (Table 1, entries 2 and 3, respectively). Single-crystal X-ray analysis of compound 2a confirmed that the vicinal aromatic rings were in the cis orientation (Table 2, entry $2 \mathrm{a}$ ). Other Lewis acids failed even after prolonged reaction times (Table 1 , entries 1 and 4-9). Generally basic conditions were not efficient in promoting the cyclization; only $\mathrm{NaH}$ in THF as a solvent successfully afforded $2 \mathrm{a}$ in $45 \%$ yield (Table1, entry 10 ). Investigating the loading of $\mathrm{BBr}_{3}$ showed that 1.5 equiv of $\mathrm{BBr}_{3}$ is sufficient to reach full conversion within $3 \mathrm{~h}$ and produce a yield of $80 \%$ (Table 1 , entry 11). Higher loadings of $\mathrm{BBr}_{3}(2-3$ equiv) resulted in a significant decrease in the level of formation of $2 a$, and the polycyclization reaction of $\mathbf{1 a}$ afforded tricyclic compound 3 (Table 1, entries 12 and 13). ${ }^{58}$ At substoichiometric loadings of $\mathrm{BBr}_{3}$, the cyclization reaction does not proceed efficiently. For instance, in the presence of 0.25 equiv of $\mathrm{BBr}_{3}$, the reaction reaches $25 \%$ conversion and stops (Table 1 , entry 14 ). The reaction performs best in chlorinated solvents such as dichloromethane and chloroform; additionally, toluene is shown to promote the reaction efficiently (Table 1 , entries 11,15 , and 16).

With our optimized conditions in hand, we first decided to verify the effectiveness of the reaction on a larger scale. It was shown that the reaction of $1 \mathrm{a}$ on a $1 \mathrm{mmol}$ scale proceeds with
Table 2. Substrates in $\mathrm{BBr}_{3}$-Promoted Intramolecular Cyclization of $1 \mathrm{a}-\mathrm{s}^{a}$

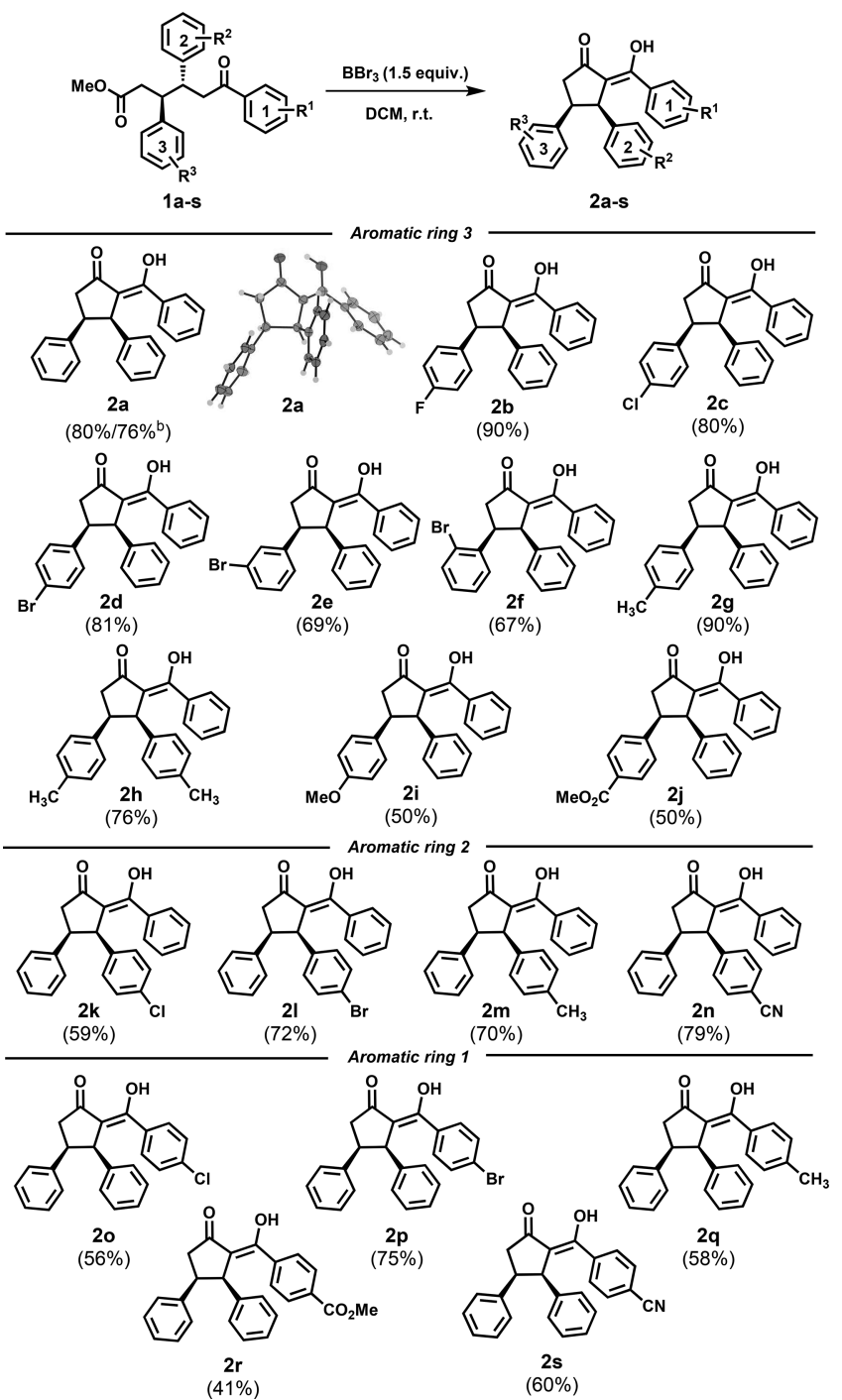

${ }^{a}$ Reactions were carried out on a $0.25 \mathrm{mmol}$ scale of $\mathbf{1}$ in DCM (3 $\mathrm{mL})$ in a sealed reaction vessel at room temperature. ${ }^{b} 1 \mathrm{mmol}$ reaction scale.

only small decrease in the yield and the corresponding compound 2 a could be isolated in $76 \%$ yield. Next, we turned our attention to the scope of the reaction (Table 2). The effect of aromatic ring 3 (Ar3) on the course of cyclization was investigated first. This revealed that the reaction tolerates a broad scope of substituents. For example, OTHOs substituted with halogens in the para position delivered the corresponding products $\mathbf{2} \mathbf{b}-\mathbf{d}$ in good yields $(81-90 \%)$. Similarly, OTHOs with a sterically demanding bromine in the meta and ortho positions on Ar3 could also be employed in the cyclization reaction. However, a decrease in the yield could be observed for compounds 2 e and 2 f (69\% and 67\%, respectively) compared to that para-substituted derivative $2 \mathrm{~d}$ (81\%). Substrates with an electron rich Ar3 are tolerated by the reaction, as well. For instance, OTHO with a methyl group at the para position on $\mathrm{Ar} 3$ provides the corresponding product $2 \mathrm{~g}$ in high yield (90\%). A slight decrease in the yield was observed when OTHO substituted with methyl groups at both Ar2 and Ar3 was used. Corresponding derivative $\mathbf{2 h}$ was 
isolated in a good yield (76\%). Introduction of a methoxy group at the para position of Ar3 decreased the yield of the corresponding product $2 \mathrm{i}$ to $50 \%$. The lower yield can be explained by the formation of a demethylated OTHO $\left(\mathbf{1 i}^{\prime}\right)$ as a side product in $25 \%$ yield. The influence of a strong electronwithdrawing group was also verified, and $\mathrm{BBr}_{3}$ applied on OTHO substituted with a methyl ester group at the para position of Ar3 led to the formation of product $2 \mathrm{j}$ in $50 \%$ yield. Next, we investigated the effect of aromatic ring $2(\operatorname{Ar} 2)$ in the cyclization reaction. The results showed lower reactivity compared to that of OTHOs substituted at Ar3. This was demonstrated on OTHOs substituted with chlorine or bromine at the para position on Ar2 where the corresponding diketones $\mathbf{2 k}$ and $2 \mathbf{l}$ could be isolated in $59 \%$ and $72 \%$ yields, respectively. OTHO substituted with a methyl at the para position of Ar2 led to the formation of $2 \mathrm{~m}$; however, the isolated yield was lower (70\%) than that of derivative $\mathbf{2 g}$ (90\%). Also, the effect of the electron-withdrawing cyano group at the para position of OTHO on Ar2 was successfully investigated, isolating the corresponding product 2 n in $79 \%$ yield. Finally, substituent effects at Ar1 of the OTHOs were investigated. Both $p$-chloro and $p$-bromo are well tolerated, and the corresponding diketones $2 \mathrm{o}$ and $\mathbf{2 p}$ were isolated in good yields $(56-75 \%)$. A comparable result was obtained with OTHO substituted with a methyl group yielding derivative $\mathbf{2 q}$ in $58 \%$ yield. In addition, OTHOs substituted with electronwithdrawing methyl ester and cyano groups at the para position on Arl were successfully employed in the cyclization reaction; however, the yields of corresponding compounds $\mathbf{2 r}$ and 2 s were lower ( $41 \%$ and $60 \%$, respectively) than those of derivatives $\mathbf{2 j}$ and $\mathbf{2 n}$. Notably, the ester group and the cyano group can be readily transferred to carboxylic acid and amide or amine, respectively, and thus, these substrates open up for the preparation of potentially biologically active compounds with hydrogen bond donor and acceptor capabilities. Expanding the scope by replacing the aryl moieties with an alkyl group is difficult as the synthesis of $\mathbf{1}$ is not compatible with alkyl substituents.

Mechanistically, we propose that the reaction starts with enolization of OTHO 1 promoted by $\mathrm{BBr}_{3}$ leading to intermediate I (Scheme 1). In the next step, the ester carbonyl is intramolecularly activated by boron leading to the borontethered species (II). In intermediate II, the enol reacts with the ester in an intramolecular fashion, resulting in boroncontaining cyclic transition intermediate III. ${ }^{59}$ At the end of the reaction, an excess of isopropanol is added to quench

Scheme 1. Proposed Mechanism of Intramolecular Cyclization of OTHOs (1) to Diketo Compounds 2

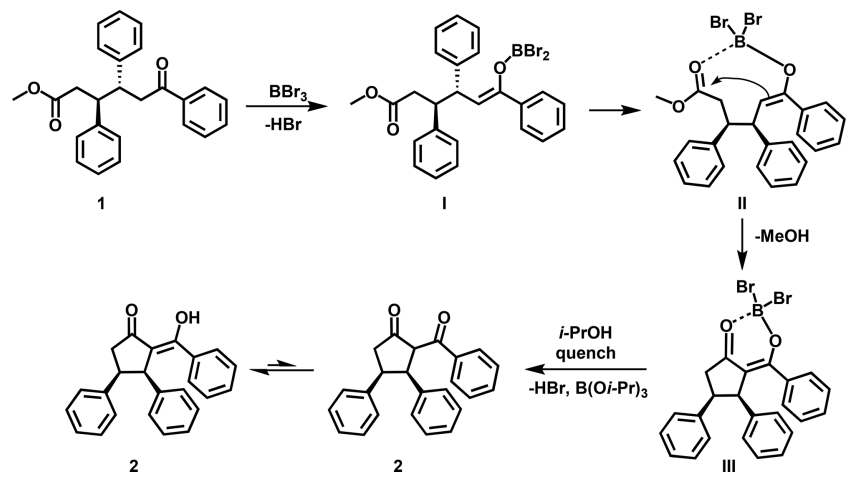

intermediate III, generating compound $\mathbf{2}$, which is stabilized in the enol form.

To demonstrate the versatility of this methodology, we used 2a as a building block in various transformations (Scheme 2).

Scheme 2. Synthetic Transformation of 2a into Different Derivatives $4-12^{a}$

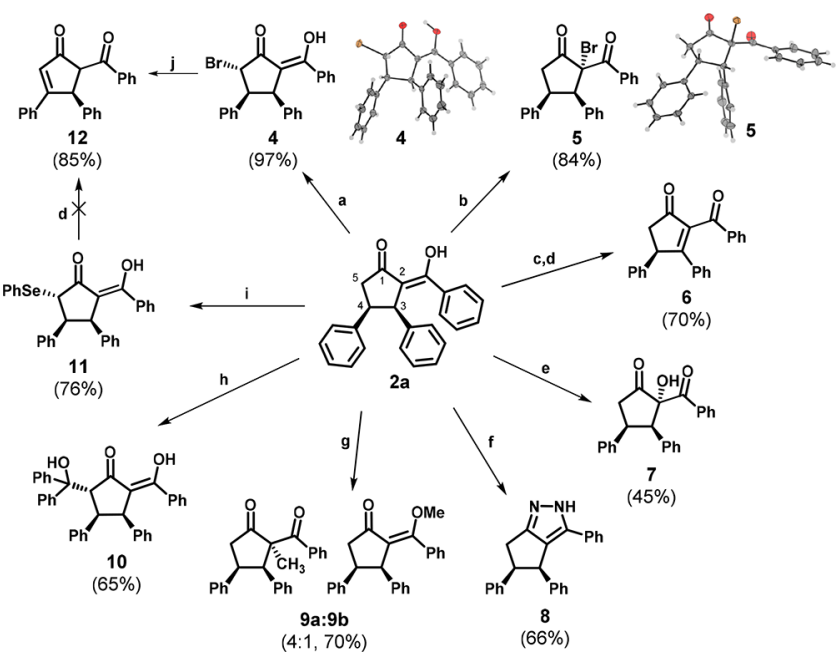

${ }^{a}$ Conditions: (a) pyridinium tribromide, DCM, rt; (b) NBS, DCM, $\mathrm{rt}$; c) $\mathrm{N}$-(phenylseleno)phthalimide; DCM, rt; (d) $\mathrm{H}_{2} \mathrm{O}_{2}$, EtAOc, rt; (e) $m$-CPBA, $\mathrm{NaHCO}_{3}, \mathrm{DCM}, \mathrm{rt}$; (f) $\mathrm{N}_{2} \mathrm{H}_{4}, \mathrm{EtOH}$, reflux; (g) $\mathrm{K}_{2} \mathrm{CO}_{3}$, MeI, DMF, $60^{\circ} \mathrm{C}$; (h) DIPA, $n$-BuLi, benzophenone, $0{ }^{\circ} \mathrm{C}$ to rt; (i) DIPA, $n$-BuLi, $N$-(phenylseleno)phthalimide, DCM, $0{ }^{\circ} \mathrm{C}$ to rt; (j) DABCO, THF, rt.

At first, we focused on bromination reactions. When 2a was subjected to $N$-bromosuccinimide, ${ }^{60} \alpha$-bromo derivative 5 was isolated in $84 \%$ yield as a single diastereoisomer. Interestingly, when 2 a was treated with pyridinium tribromide, $\delta$-brominated regioisomer 4 was isolated in high yield (97\%). This bromination strategy offers a route toward compounds that can be used as building blocks in, for example, the Reformatsky type cyanation reaction mediated by samarium salts ${ }^{61}$ or in organocatalytic cyclopropanation reactions. ${ }^{62}$ Remarkably, compound $\mathbf{4}$ was like derivative $\mathbf{5}$ isolated as a single diastereoisomer with an anti orientation of bromine and both aromatic rings. This relative configuration of substituents on the cyclopentanone ring was confirmed by X-ray diffraction of both derivatives $\mathbf{4}$ and $\mathbf{5}$ (see the Supporting Information).

Apart from bromination, we also showed that $2 \mathrm{a}$ can be regioselectively substituted with phenylselenium on either C-2 or C-5 of cyclopentanone based on the used conditions. When 2a was subjected to $N$-(phenylseleno)phthalimide in the presence of 2 equiv of LDA, prepared in situ, selenylated product 11 was isolated in $76 \%$ yield as a single diastereoisomer, whereas selenylation with $\mathrm{N}$-(phenylseleno)phthalimide without a base led to the selenylation on C-2 of 2a. The corresponding intermediate was unstable upon isolation, but after the crude mixture had been treated with hydrogen peroxide, the elimination reaction took place and unsaturated derivative 6 was isolated in $70 \%$ overall yield. The same reaction conditions were also used on compound 11 . Unfortunately, elimination did not proceed, and decomposition of the starting material was observed. On the contrary, corresponding unsaturated derivative $\mathbf{1 2}$ was isolated in good yield (85\%) after treatment of 4 with 1,4-diazabicyclo[2.2.2]- 
octane (DABCO) in THF at room temperature. Furthermore, diastereoselective hydroxylation of $2 \mathrm{a}$ was developed in the presence of $m$-CPBA and hydroxylated diketone 7 could be isolated in $45 \%$ yield. Next, the possibility of $\mathrm{C}-\mathrm{C}$ bond formation on C-2 and C-5 of $\mathbf{2 a}$ was investigated. When $\mathbf{2 a}$ was treated with iodomethane in the presence of potassium carbonate, methylated product 9 was isolated as a single diastereoisomer in a good yield of $70 \%$, however, as a mixture of regioisomers (4:1 keto:enol). On the contrary, when 2 equiv of in situ-generated LDA was used in the presence of $2 a$ and benzophenone as an electrophile, ${ }^{63}$ the corresponding product 10 was isolated in $65 \%$ yield as a single diastereomer. These examples nicely illustrate the influence of both cis-oriented aromatic rings on stereoselective addition of electrophiles to both $\mathrm{C}-2$ and $\mathrm{C}-5$ to the unhindered side of the cyclic keto ester. We were also able to show that 2 a can be transformed into pyrazole derivative 8 upon being treated with hydrazine hydrate in ethanol under reflux conditions. ${ }^{64}$

To validate whether our diastereoselective synthesis of cisvic-diphenylethylene dicarbonyl compounds could be useful for the synthesis of compounds that are structurally similar to rocaglamide or silvestrol, a superimposition was made between compound $2 \mathrm{t}$ and rocaglamide. As it turns out, the superimposition shows an unambiguous match between both cisoriented neighboring Ar of $\mathbf{2 t}$ and the rocaglamide aromatic rings (Figure 2). This provides evidence that the geometry of

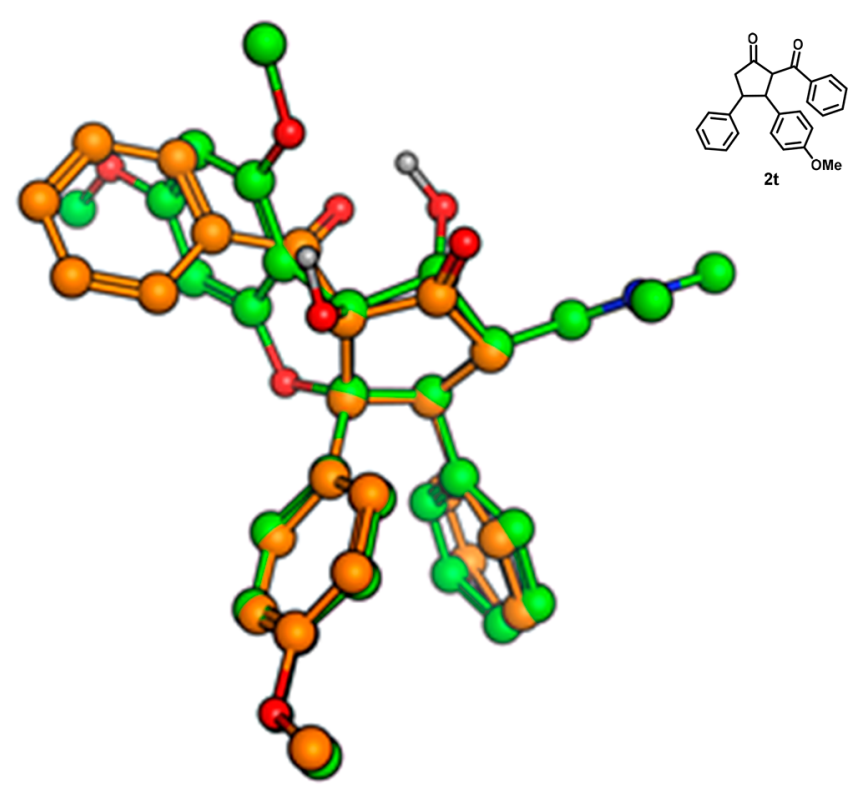

Figure 2. Superimposition of the global minima conformation of rocaglamide (green) and a low-energy conformation $(\Delta E=0.7 \mathrm{kcal}$ $\mathrm{mol}^{-1}$ ) of $2 \mathrm{t}$ (orange).

the diphenylethylene moiety found in the series of compound 2 mimics the diphenylethylene found in natural products such as rocaglamide and silvestrol.

\section{CONCLUSION}

We have developed an intramolecular cyclization reaction of 1,6-ketoesters using boron tribromide as a Lewis acid promoter. This methodology provides straightforward access to 1,3-diketo compounds with a cyclopentane moiety having a cis-oriented vicinal diphenylethylene that is unique and difficult to obtain by known synthetic methods. We have also shown that the defined orientation of both vicinal aromatic rings has a directing effect on stereoselectivity in subsequent substitution reactions with bromine, selenium, or a carbon electrophile on C-2 or C-5 of the 1,3-diketones (Scheme 2). Also, regioselective elimination procedures were developed on the basis of the substitution at $\mathrm{C}-2$ or $\mathrm{C}-5$ providing access to two types of $\alpha, \beta$-unsaturated carbonyl compounds. Moreover, successful transformation of a 1,3-diketo compound to a heterocyclic pyrazole derivative has been demonstrated showing the importance of 1,3-diketones as potential building blocks for druglike architectures. Additionally, the matching superimposition of $\mathbf{2 t}$ and rocaglamide indicates that the substructure found in compound $\mathbf{2}$ is a good starting point for the synthesis of a molecular library for probing biological activity. Compounds including cis-vic-diphenylethylene-oriented motifs such as rocaglamide and silverstrol have attracted considerable pharmaceutical interest, e.g., in cancer treatment and as antiviral agents. ${ }^{21,36-38}$ The synthesis protocol presented here can easily be fine-tuned to achieve a wanted pharmacological profile and thus opens up new opportunities to further explore new analogues for the therapies mentioned above.

\section{EXPERIMENTAL SECTION}

Molecular Modeling. To verify the hypothesis that the aromatic rings in $2 t$ can mimic the corresponding rings in rocaglamide, molecular mechanics calculations were used. A conformational search was performed on both compounds using the Amber10:EHT force field, the Born aqueous solvation model, and the LowModeMD search methodology. The standard settings were applied, and the energy cutoff was set to $5 \mathrm{kcal} / \mathrm{mol}$ from their global minima. An ensemble of conformations of the two compounds ( 24 of rocaglamide and 14 of $2 t$ ) was achieved, and these were crosswise superimposed (336 combinations). The conclusion was that superimposition of $\mathbf{2 t}$ mimics rocaglamide very well, and among the top-scoring combinations were the global minima of rocaglamide and a lowenergy conformation of $2 \mathrm{t}$, i.e., $\Delta E=0$ and $0.7 \mathrm{kcal} \mathrm{mol}^{-1}$ (Figure 2). The superimposition queries were aromatic rings 2 and 3 , the methoxy oxygen, and C-3 and C-4 in the five-membered ring (see Scheme 2 for numbering). All molecular calculations were performed using tools implemented in MOE modeling software. ${ }^{65}$

General Experimental Information. Column chromatography was performed on automated column chromatography Biotage Isolera Spektra One with Biotage SNAP-10g KP-sil columns. Thin layer chromatography (TLC) was performed on Merck TLC plates precoated with silica gel $60 \mathrm{~F} 254$ (Art 5715, $0.25 \mathrm{~mm}$ ) and visualized with ultraviolet light $(254 \mathrm{~nm})$. The ${ }^{1} \mathrm{H}$ NMR $(400 \mathrm{MHz})$ and ${ }^{13} \mathrm{C}$ NMR $(101 \mathrm{MHz})$ spectra were recorded on a Varian 400 instrument, and ${ }^{19} \mathrm{~F}$ NMR $(470 \mathrm{MHz})$ spectra were recorded on a Varian 500 spectrometer. The chemical shifts are reported in parts per million $(\delta)$ relative to the residual solvent peak $\mathrm{CDCl}_{3}:{ }^{1} \mathrm{H}$ NMR at $\delta 7.26$ and ${ }^{13} \mathrm{C}$ NMR at $\delta$ 77.16. Coupling constants $(J)$ are reported in hertz. Infrared (IR) spectra were recorded on a PerkinElmer series FT-IR spectrometer and are reported in wavenumbers $\left(\mathrm{cm}^{-1}\right)$. Melting points were recorded on a Stuart Scientific Melting Point SMP1 instrument. Gas chromatographic studies were performed using an Agilent 7820A instrument equipped with a flame ionization detector and an Agilent HP-5 19091J-413 column. Crystallographic data were obtained using a Bruker D8 VENTURE Kappa Duo PHOTONIII instrument. The 1-ethyl-3-methylimidazolium acetate (EMIMAc) was purchased from Sigma-Aldrich (Stockholm, Sweden), produced by BASF $\geq 95 \%$, and dried in vacuo with heating prior to use. All other solvents and reagents were purchased from commercial sources and used without further treatments.

Synthesis of the Starting Material, OTHOs (1). OTHOs $\mathbf{1 a}-\mathbf{d}, \mathbf{1 g}$, $1 \mathrm{i}-\mathrm{l}, 1 \mathrm{n}, 1 \mathrm{o}, 1 \mathrm{e}, 1 \mathrm{f}, 1 \mathrm{~h}, 1 \mathrm{~m}$, and $1 \mathrm{p}-\mathrm{s}$ were synthesized according to previously published procedures.
52,58 
Methyl 4-(4-Cyanophenyl)-6-oxo-3,4-diphenylhexanoate (1n). Isolated as a white solid (450 mg, 53\% yield); mp $167-169{ }^{\circ} \mathrm{C}$ (from $\mathrm{MeOH}) ;{ }^{1} \mathrm{H}$ NMR (400 MHz, $\left.\mathrm{CDCl}_{3}\right) \delta 7.67-7.57(\mathrm{~m}, 4 \mathrm{H})$, $7.52-7.42(\mathrm{~m}, 3 \mathrm{H}), 7.39-7.22(\mathrm{~m}, 7 \mathrm{H}), 3.70\left(\mathrm{td}, J=10.8 \mathrm{~Hz}, J^{\prime}=\right.$ $3.2 \mathrm{~Hz}, 1 \mathrm{H}), 3.48-3.39(\mathrm{~s}, 4 \mathrm{H}), 3.28\left(\mathrm{dd}, J=17.2 \mathrm{~Hz}, J^{\prime}=10.5 \mathrm{~Hz}\right.$ $1 \mathrm{H}), 2.96\left(\mathrm{dd}, J=17.3 \mathrm{~Hz}, J^{\prime}=3.2 \mathrm{~Hz}, 1 \mathrm{H}\right), 2.50\left(\mathrm{dd}, J=15.6 \mathrm{~Hz}, J^{\prime}\right.$ $=10.2 \mathrm{~Hz}, 1 \mathrm{H}), 2.34\left(\mathrm{dd}, J=15.6 \mathrm{~Hz}, J^{\prime}=4.5 \mathrm{~Hz}, 1 \mathrm{H}\right) ;{ }^{13} \mathrm{C}\left\{{ }^{1} \mathrm{H}\right\}$ NMR $\left(101 \mathrm{MHz}, \mathrm{CDCl}_{3}\right) \delta 197.8,172.2,148.4,141.3,136.7,133.3$, 132.6 (2C), 129.4 (2C), 129.1 (2C), 128.7 (2C), 128.2 (2C), 127.9 (2C), 127.6, 118.9, 111.0, 51.7, 47.4, 46.7, 43.4, 39.8; ATR-FTIR $\nu$ 2951, 2228, 1726, 1718, 1679, 1609, 1597, 1436, 1237, $1157 \mathrm{~cm}^{-1}$; HRMS (ESI) $m / z$ calcd for $\mathrm{C}_{26} \mathrm{H}_{24} \mathrm{NO}_{3}[\mathrm{M}+\mathrm{H}]^{+} 398.1751$, found 398.1751.

General Procedure for the $\mathrm{BBr}_{3}$-Promoted Synthesis of 2. To a flask equipped with a magnetic stir bar were added OTHO 1a-s ( $0.25 \mathrm{mmol}, 1$ equiv) and DCM $(3 \mathrm{~mL})$. The solution was stirred at room temperature under nitrogen and syringed with a solution of $\mathrm{BBr}_{3}(375 \mu \mathrm{L}, 1 \mathrm{M}$ in $\mathrm{DCM}, 1.5$ equiv). The mixture was stirred until full conversion was reached (monitored by TLC), then the reaction quenched with $i-\operatorname{PrOH}(2 \mathrm{~mL})$, and the mixture evaporated to dryness. The crude material was separated by flash chromatography with a petroleum ether/EtOAc mixture, yielding the corresponding compound $\mathbf{2 a}-\mathbf{s}$.

Procedure for the $\mathrm{BBr}_{3}$-Promoted Synthesis of $2 \mathrm{a}$ on a $1 \mathrm{mmol}$ Scale. To a flask equipped with a magnetic stir bar were added OTHO 1a $(0.372 \mathrm{mmol}, 1$ equiv) and DCM $(12 \mathrm{~mL})$. The solution was stirred at room temperature under nitrogen and syringed with a solution of $\mathrm{BBr}_{3}$ ( $1.5 \mathrm{~mL}, 1 \mathrm{M}$ in DCM, 1.5 equiv). The mixture was stirred until full conversion was reached (monitored by TLC), then the reaction quenched with $i-\mathrm{PrOH}(8 \mathrm{~mL})$, and the mixture evaporated to dryness. The crude material was separated by flash chromatography in a petroleum ether/EtOAc mixture yielding corresponding compound $2 \mathrm{a}$ as a yellow solid (204 mg, 76\%).

(2Z)-2-[Hydroxy(phenyl)methylidene]-3,4-diphenylcyclopentan1-one (2a). Purified in 92:8 petroleum ether/EtOAc; $68 \mathrm{mg}$ yield as a yellow solid (80\%); mp $121-123{ }^{\circ} \mathrm{C}$ (from $\mathrm{MeOH}$ ); 1:10 keto:enol. Enol: ${ }^{1} \mathrm{H}$ NMR $\left(400 \mathrm{MHz}, \mathrm{CDCl}_{3}\right) \delta 7.55-7.49(\mathrm{~m}, 2 \mathrm{H}), 7.40-7.34$ $(\mathrm{m}, 1 \mathrm{H}), 7.28-7.24(\mathrm{~m}, 3 \mathrm{H}), 7.12-7.06(\mathrm{~m}, 6 \mathrm{H}), 6.78-6.74(\mathrm{~m}$, $3 \mathrm{H}), 4.36(\mathrm{~d}, J=7.4 \mathrm{~Hz}, 1 \mathrm{H}), 3.87\left(\mathrm{dt}, J=14.0 \mathrm{~Hz}, J^{\prime}=7.2 \mathrm{~Hz}, 1 \mathrm{H}\right)$, 3.09 (dd, $\left.J=17.4 \mathrm{~Hz}, J^{\prime}=13.5 \mathrm{~Hz}, 1 \mathrm{H}\right), 2.62\left(\mathrm{dd}, J=17.3 \mathrm{~Hz}, J^{\prime}=\right.$ $7.1 \mathrm{~Hz}, 1 \mathrm{H}) ;{ }^{13} \mathrm{C}\left\{{ }^{1} \mathrm{H}\right\}$ NMR $\left(101 \mathrm{MHz}, \mathrm{CDCl}_{3}\right) \delta 209.3,170.8$, $140.5,138.9,133.9,131.2,128.6$ (2C), 128.4 (2C), 128.3 (2C), 128.2 (4C), 127.9 (2C), 126.8, 126.7, 113.3, 52.0, 48.3, 39.8. Keto: ${ }^{1} \mathrm{H}$ NMR $\left(400 \mathrm{MHz}, \mathrm{CDCl}_{3}\right) \delta 8.04-7.96(\mathrm{~m}, 2 \mathrm{H}), 7.62-7.56(\mathrm{~m}, 1 \mathrm{H})$, $7.50-7.48(\mathrm{~m}, 2 \mathrm{H}), 7.17-7.13(\mathrm{~m}, 3 \mathrm{H}), 7.11-7.06(\mathrm{~m}, 3 \mathrm{H}), 6.82-$ $6.76(\mathrm{~m}, 4 \mathrm{H}), 4.83(\mathrm{~d}, J=8.3 \mathrm{~Hz}, 1 \mathrm{H}), 4.57-4.44(\mathrm{~m}, 1 \mathrm{H}), 4.07(\mathrm{q}, J$ $=7.0 \mathrm{~Hz}, 1 \mathrm{H}), 3.01-2.92(\mathrm{~m}, 2 \mathrm{H}) ;{ }^{13} \mathrm{C}\left\{{ }^{1} \mathrm{H}\right\}$ NMR $(101 \mathrm{MHz}$, $\left.\mathrm{CDCl}_{3}\right) \delta 212.3,194.5,139.6,138.6,136.6,133.7,129.6,128.7$ (2C), 128.3 (2C), 128.2 (3C), 128.1 (4C), 127.0, 126.9, 61.2, 51.1, 45.5, 44.8; ATR-FTIR $\nu 1758,1642,1608,1593,1570,1357,1268,1222$, $1146,1099 \mathrm{~cm}^{-1}$; HRMS (ESI) $\mathrm{m} / z$ calcd for $\mathrm{C}_{24} \mathrm{H}_{21} \mathrm{O}_{2}[\mathrm{M}+\mathrm{H}]^{+}$ 341.1537, found 341.1536 .

(2Z)-4-(4-Fluorophenyl)-2-[hydroxy(phenyl)methylidene]-3-phenylcyclopentan-1-one (2b). Purified in 99:1 to 97:3 petroleum ether/EtOAc; $81 \mathrm{mg}$ yield as a thick orange oil (90\%); 1:10 keto:enol. Enol: ${ }^{1} \mathrm{H}$ NMR $\left(400 \mathrm{MHz}, \mathrm{CDCl}_{3}\right) \delta 7.55-7.51(\mathrm{~m}, 2 \mathrm{H}), 7.41-7.34$ $(\mathrm{m}, 1 \mathrm{H}), 7.26(\mathrm{~s}, 2 \mathrm{H}), 7.17-7.09(\mathrm{~m}, 3 \mathrm{H}), 6.83-6.66(\mathrm{~m}, 6 \mathrm{H}), 4.33$ $(\mathrm{d}, J=7.4 \mathrm{~Hz}, 1 \mathrm{H}), 3.85\left(\mathrm{dt}, J=14.0 \mathrm{~Hz}, J^{\prime}=7.2 \mathrm{~Hz}, 1 \mathrm{H}\right), 3.02(\mathrm{dd}, J$ $\left.=17.3 \mathrm{~Hz}, J^{\prime}=13.5 \mathrm{~Hz}, 1 \mathrm{H}\right), 2.62\left(\mathrm{dd}, J=17.3 \mathrm{~Hz}, J^{\prime}=7.1 \mathrm{~Hz}, 1 \mathrm{H}\right)$; ${ }^{13} \mathrm{C}\left\{{ }^{1} \mathrm{H}\right\}$ NMR $\left(101 \mathrm{MHz}, \mathrm{CDCl}_{3}\right) \delta 208.9,171.1,161.7$ (d, $J=245.2$ $\mathrm{Hz}), 140.4,134.7(\mathrm{~d}, J=3.1 \mathrm{~Hz}), 133.9,131.3,129.6(\mathrm{~d}, J=8.0 \mathrm{~Hz}$, 2C), $128.6(2 \mathrm{C}), 128.4(6 \mathrm{C}), 126.9,114.8(\mathrm{~d}, J=21.2 \mathrm{~Hz}, 2 \mathrm{C})$, 113.1, 52.0, 47.6, 40.1; ${ }^{19} \mathrm{~F}$ NMR $\left(470 \mathrm{MHz}, \mathrm{CDCl}_{3}\right) \delta-115.9$ (ddd, $\left.J=13.7 \mathrm{~Hz}, J^{\prime}=8.3 \mathrm{~Hz}, J^{\prime \prime}=5.4 \mathrm{~Hz}\right)$. Keto: ${ }^{1} \mathrm{H}$ NMR $(400 \mathrm{MHz}$, $\left.\mathrm{CDCl}_{3}\right) \delta 8.07-7.96(\mathrm{~m}, 2 \mathrm{H}), 7.62-7.57(\mathrm{~m}, 1 \mathrm{H}), 7.50-7.45(\mathrm{~m}$, $2 \mathrm{H}), 7.28-7.24(\mathrm{~m}, 1 \mathrm{H}), 7.17-7.09(\mathrm{~m}, 2 \mathrm{H}), 6.88-6.74(\mathrm{~m}, 6 \mathrm{H})$, $4.82(\mathrm{~d}, J=7.7 \mathrm{~Hz}, 1 \mathrm{H}), 4.46(\mathrm{t}, J=7.3 \mathrm{~Hz}, 1 \mathrm{H}), 4.08(\mathrm{q}, J=7.2 \mathrm{~Hz}$, $1 \mathrm{H}), 2.93(\mathrm{~d}, J=7.8 \mathrm{~Hz}, 2 \mathrm{H}) ;{ }^{13} \mathrm{C}\left\{{ }^{1} \mathrm{H}\right\} \operatorname{NMR}\left(101 \mathrm{MHz}, \mathrm{CDCl}_{3}\right) \delta$ 212.0, 194.4, $161.8(\mathrm{~d}, J=245.6 \mathrm{~Hz}), 138.6,136.4,135.3(\mathrm{~d}, J=3.3$ $\mathrm{Hz}), 133.9,133.9,129.7$ (2C), 129.5 (d, J = 8.0 Hz, 2C), 128.8 (2C),
128.2 (2C), 127.2, 115.1 (d, $J=21.2 \mathrm{~Hz}, 2 \mathrm{C}), 61.4,51.1,44.9,44.6$; ${ }^{19} \mathrm{~F} \mathrm{NMR}\left(470 \mathrm{MHz}, \mathrm{CDCl}_{3}\right) \delta-115.8\left(\mathrm{tt}, J=8.5 \mathrm{~Hz}, J^{\prime}=5.5 \mathrm{~Hz}\right)$; ATR-FTIR $\nu 2905,1746,1636,1592,1565,1510,1492,1448,1352$, $1219,1150,1096 \mathrm{~cm}^{-1}$; HRMS (ESI) $\mathrm{m} / z$ calcd for $\mathrm{C}_{24} \mathrm{H}_{20} \mathrm{FO}_{2}[\mathrm{M}+$ $\mathrm{H}]^{+}$359.1442, found 359.1437.

(2Z)-4-(4-Chlorophenyl)-2-[hydroxy(phenyl)methylidene]-3-phenylcyclopentan-1-one (2c). Purified in 98:2 to 97:3 petroleum ether/ EtOAc; $74 \mathrm{mg}$ yield as a thick orange oil (80\%); 1:10 keto:enol. Enol: ${ }^{1} \mathrm{H}$ NMR $\left(400 \mathrm{MHz}, \mathrm{CDCl}_{3}\right) \delta$ 7.57-7.47 (m, 2H), 7.43-7.34 (m, $1 \mathrm{H}), 7.26(\mathrm{~s}, 2 \mathrm{H}), 7.18-7.11(\mathrm{~m}, 3 \mathrm{H}), 7.09-7.02(\mathrm{~m}, 2 \mathrm{H}), 6.82-$ $6.76(\mathrm{~m}, 2 \mathrm{H}), 6.67(\mathrm{~d}, J=8.4 \mathrm{~Hz}, 2 \mathrm{H}), 4.34(\mathrm{~d}, J=7.4 \mathrm{~Hz}, 1 \mathrm{H}), 3.83$ $\left(\mathrm{dt}, J=14.0 \mathrm{~Hz}, J^{\prime}=7.2 \mathrm{~Hz}, 1 \mathrm{H}\right), 3.03\left(\mathrm{dd}, J=17.3 \mathrm{~Hz}, J^{\prime}=13.4 \mathrm{~Hz}\right.$, $1 \mathrm{H}), 2.61\left(\mathrm{dd}, J=17.3 \mathrm{~Hz}, J^{\prime}=7.1 \mathrm{~Hz}, 1 \mathrm{H}\right) ;{ }^{13} \mathrm{C}\left\{{ }^{1} \mathrm{H}\right\}$ NMR $(101$ $\left.\mathrm{MHz}, \mathrm{CDCl}_{3}\right) \delta 208.7,171.1,140.3,137.6,133.9,132.5,131.4,129.5$ (2C), 128.6 (2C), 128.5 (2C), 128.4 (4C), 128.1 (2C), 127.0, 113.1, 51.8, 47.7, 39.9. Keto: ${ }^{1} \mathrm{H}$ NMR $\left(400 \mathrm{MHz}, \mathrm{CDCl}_{3}\right) \delta 8.02(\mathrm{dd}, J=$ $\left.8.4 \mathrm{~Hz}, J^{\prime}=1.3 \mathrm{~Hz}, 2 \mathrm{H}\right), 7.64-7.55(\mathrm{~m}, 1 \mathrm{H}), 7.50-7.46(\mathrm{~m}, 2 \mathrm{H})$, $7.28-7.24(\mathrm{~m}, 3 \mathrm{H}), 7.16-7.10(\mathrm{~m}, 4 \mathrm{H}), 6.82-6.76(\mathrm{~m}, 2 \mathrm{H}), 6.76-$ $6.71(\mathrm{~m}, 2 \mathrm{H}), 4.82(\mathrm{~d}, J=7.6 \mathrm{~Hz}, 1 \mathrm{H}), 4.47(\mathrm{t}, J=7.3 \mathrm{~Hz}, 1 \mathrm{H}), 4.08$ $(\mathrm{q}, J=7.3 \mathrm{~Hz}, 1 \mathrm{H}), 2.92(\mathrm{~d}, J=7.6 \mathrm{~Hz}, 2 \mathrm{H}) ;{ }^{13} \mathrm{C}\left\{{ }^{1} \mathrm{H}\right\}$ NMR $(101$ $\left.\mathrm{MHz}, \mathrm{CDCl}_{3}\right) \delta 211.8,194.3,138.4,138.1,136.4,132.7,131.4,129.7$ (2C), $129.4(2 \mathrm{C}), 128.8(2 \mathrm{C}), 128.5(2 \mathrm{C}), 128.3(2 \mathrm{C}), 128.2(2 \mathrm{C})$, 127.3, 61.5, 51.0, 45.0, 44.4; ATR-FTIR $\nu$ 3026, 1743, 1595, 1570, $1493,1447,1362,1266,1226,1091,1016 \mathrm{~cm}^{-1}$; HRMS (ESI) $\mathrm{m} / z$ calcd for $\mathrm{C}_{24} \mathrm{H}_{20} \mathrm{ClO}_{2}[\mathrm{M}+\mathrm{H}]^{+} 375.1146$, found 375.1160 .

(2Z)-4-(4-Bromophenyl)-2-[hydroxy(phenyl)methylidene]-3-phenylcyclopentan-1-one (2d). Purified in 98:2 to 97:3 petroleum ether/EtOAc; $85 \mathrm{mg}$ yield as a thick orange oil $(81 \%) ; 1: 10$ keto:enol. Enol: ${ }^{1} \mathrm{H}$ NMR $\left(400 \mathrm{MHz}, \mathrm{CDCl}_{3}\right) \delta 7.55-7.49(\mathrm{~m}, 2 \mathrm{H}), 7.42-7.35$ (m, 1H), 7.29-7.19 (m, 4H), 7.17-7.10 (m, 3H), 6.81-6.77 (m, $2 \mathrm{H}), 6.65-6.57(\mathrm{~m}, 2 \mathrm{H}), 4.35(\mathrm{~d}, J=7.4 \mathrm{~Hz}, 1 \mathrm{H}), 3.81(\mathrm{dt}, J=14.0$ $\left.\mathrm{Hz}, J^{\prime}=7.2 \mathrm{~Hz}, 1 \mathrm{H}\right), 3.02\left(\mathrm{dd}, J=17.3 \mathrm{~Hz}, J^{\prime}=13.5 \mathrm{~Hz}, 1 \mathrm{H}\right), 2.61$ $\left(\mathrm{dd}, J=17.3 \mathrm{~Hz}, J^{\prime}=7.1 \mathrm{~Hz}, 1 \mathrm{H}\right) ;{ }^{13} \mathrm{C}\left\{{ }^{1} \mathrm{H}\right\}$ NMR $(101 \mathrm{MHz}$, $\left.\mathrm{CDCl}_{3}\right) \delta 208.7,171.1,140.2,138.1,133.9,131.3,131.0$ (2C), 129.9 (2C), 128.6 (2C), 128.5 (2C), 128.4 (4C), 127.0, 120.7, 113.1, 51.8, 47.8, 39.9. Keto: ${ }^{1} \mathrm{H}$ NMR $\left(400 \mathrm{MHz}, \mathrm{CDCl}_{3}\right) \delta 8.07-7.99(\mathrm{~m}, 2 \mathrm{H})$, 7.62-7.57 (m, 1H), 7.50-7.46 (m, 1H), 7.26 (s, 1H), 7.17-7.09 (m, $4 \mathrm{H}), 6.82-6.75(\mathrm{~m}, 2 \mathrm{H}), 6.68(\mathrm{~d}, J=8.4 \mathrm{~Hz}, 2 \mathrm{H}), 4.82(\mathrm{~d}, J=7.6$ $\mathrm{Hz}, 1 \mathrm{H}), 4.47(\mathrm{t}, J=7.3 \mathrm{~Hz}, 1 \mathrm{H}), 4.06(\mathrm{q}, J=7.3 \mathrm{~Hz}, 1 \mathrm{H}), 2.95-2.89$ $(\mathrm{m}, 2 \mathrm{H}) ;{ }^{13} \mathrm{C}\left\{{ }^{1} \mathrm{H}\right\}$ NMR $\left(101 \mathrm{MHz}, \mathrm{CDCl}_{3}\right) \delta 211.7,194.2,138.6$, 138.4, 136.4, 133.9, 131.3 (2C), 129.7 (4C), 128.8 (2C), 128.5 (2C), 128.1 (2C), 127.3, 120.8, 61.4, 50.9, 45.1, 44.3; ATR-FTIR $\nu$ 3028, $1746,1596,1567,1490,1365,1263,1225,1077,1011 \mathrm{~cm}^{-1}$; HRMS (ESI) $m / z$ calcd for $\mathrm{C}_{24} \mathrm{H}_{20} \mathrm{BrO}_{2}[\mathrm{M}+\mathrm{H}]^{+}$419.0641, found 419.0635 .

(2Z)-4-(3-Bromophenyl)-2-[hydroxy(phenyl)methylidene]-3-phenylcyclopentan-1-one (2e). Purified in 97:3 to 95:5 petroleum ether/ EtOAc; $72 \mathrm{mg}$ yield as a thick orange oil $(69 \%) ; 1: 10$ keto:enol. Enol: ${ }^{1} \mathrm{H}$ NMR $\left(400 \mathrm{MHz}, \mathrm{CDCl}_{3}\right) \delta 7.56-7.51(\mathrm{~m}, 2 \mathrm{H}), 7.42-7.35(\mathrm{~m}$, $1 \mathrm{H}), 7.30-7.22(\mathrm{~m}, 3 \mathrm{H}), 7.16-7.13(\mathrm{~m}, 3 \mathrm{H}), 6.96-6.89(\mathrm{~m}, 2 \mathrm{H})$, 6.81-6.77 (m, 2H), 6.67-6.63 (m, 1H), $4.35(\mathrm{~d}, J=7.4 \mathrm{~Hz}, 1 \mathrm{H})$, $3.81\left(\mathrm{dt}, J=14.1 \mathrm{~Hz}, J^{\prime}=7.3 \mathrm{~Hz}, 1 \mathrm{H}\right), 3.03\left(\mathrm{dd}, J=17.3 \mathrm{~Hz}, J^{\prime}=13.4\right.$ $\mathrm{Hz}, 1 \mathrm{H}), 2.62\left(\mathrm{dd}, J=17.3 \mathrm{~Hz}, J^{\prime}=7.2 \mathrm{~Hz}, 1 \mathrm{H}\right) ;{ }^{13} \mathrm{C}\left\{{ }^{1} \mathrm{H}\right\}$ NMR $(101$ $\left.\mathrm{MHz}, \mathrm{CDCl}_{3}\right) \delta 208.5,171.2,141.5,140.2,133.8,131.5,131.4,129.9$, $129.5,128.5$ (2C), 128.4 (6C), 127.1, 126.7, 122.1, 113.0, 51.9, 48.0 39.7. Keto: ${ }^{1} \mathrm{H}$ NMR $\left(400 \mathrm{MHz}, \mathrm{CDCl}_{3}\right) \delta 8.06-8.00(\mathrm{~m}, 2 \mathrm{H})$, $7.62-7.57(\mathrm{~m}, 1 \mathrm{H}), 7.51-7.47(\mathrm{~m}, 1 \mathrm{H}), 7.29-7.22(\mathrm{~m}, 3 \mathrm{H}), 7.16-$ $7.13(\mathrm{~m}, 3 \mathrm{H}), 7.03-6.99(\mathrm{~m}, 1 \mathrm{H}), 6.83-6.77(\mathrm{~m}, 2 \mathrm{H}), 6.76-6.72$ $(\mathrm{m}, 1 \mathrm{H}), 4.82(\mathrm{~d}, J=7.1 \mathrm{~Hz}, 1 \mathrm{H}), 4.46(\mathrm{t}, J=7.1 \mathrm{~Hz}, 1 \mathrm{H}), 4.08(\mathrm{q}, J$ $=7.4 \mathrm{~Hz}, 1 \mathrm{H}), 2.92\left(\mathrm{dd}, J=7.8 \mathrm{~Hz}, J^{\prime}=1.1 \mathrm{~Hz}, 1 \mathrm{H}\right) ;{ }^{13} \mathrm{C}\left\{{ }^{1} \mathrm{H}\right\} \mathrm{NMR}$ $\left(101 \mathrm{MHz}, \mathrm{CDCl}_{3}\right) \delta 211.6,194.2,141.9,138.4,136.3,133.9,131.3$, 130.0, 129.7 (3C), 128.8 (2C), 128.4 (2C), 128.1 (2C), 127.4, 126.5, 122.4, 61.7, 51.0, 45.3, 43.9; ATR-FTIR $\nu$ 3024, 2917, 1744, 1637, $1592,1569,1494,1475,1357,1265,1225,1152,1098,1075 \mathrm{~cm}^{-1}$; HRMS (ESI) $m / z$ calcd for $\mathrm{C}_{24} \mathrm{H}_{20} \mathrm{BrO}_{2}[\mathrm{M}+\mathrm{H}]^{+} 419.0641$, found 419.0631 .

(2Z)-4-(2-Bromophenyl)-2-[hydroxy(phenyl)methylidene]-3-phenylcyclopentan-1-one (2f). Purified in 97:3 to 95:5 petroleum ether/ EtOAc; $70 \mathrm{mg}$ yield as a thick orange oil $(67 \%) ; 1: 24$ keto:enol; ${ }^{1} \mathrm{H}$ $\operatorname{NMR}\left(400 \mathrm{MHz}, \mathrm{CDCl}_{3}\right) \delta 7.62-7.49(\mathrm{~m}, 3 \mathrm{H}), 7.42-7.34(\mathrm{~m}, 1 \mathrm{H})$, 
$7.30-7.26(\mathrm{~m}, 2 \mathrm{H}), 7.12-7.04(\mathrm{~m}, 3 \mathrm{H}), 6.99-6.94(\mathrm{~m}, 1 \mathrm{H}), 6.92-$ $6.79(\mathrm{~m}, 3 \mathrm{H}), 6.41\left(\mathrm{dd}, J=7.9 \mathrm{~Hz}, J^{\prime}=1.7 \mathrm{~Hz}, 1 \mathrm{H}\right), 4.73(\mathrm{~d}, J=7.4$ $\mathrm{Hz}, 1 \mathrm{H}), 4.29$ (dt, $\left.J=14.1 \mathrm{~Hz}, J^{\prime}=7.2 \mathrm{~Hz}, 1 \mathrm{H}\right), 3.13$ (dd, $J=17.2$ $\left.\mathrm{Hz}, J^{\prime}=13.7 \mathrm{~Hz}, 1 \mathrm{H}\right), 2.59\left(\mathrm{dd}, J=17.2 \mathrm{~Hz}, J^{\prime}=7.1 \mathrm{~Hz}, 1 \mathrm{H}\right)$; ${ }^{13} \mathrm{C}\left\{{ }^{1} \mathrm{H}\right\}$ NMR $\left(101 \mathrm{MHz}, \mathrm{CDCl}_{3}\right) \delta 208.4,171.5,140.8,138.0$, $133.9,132.7,131.4,128.5$ (2C), 128.4 (5C), 128.3, 128.0 (2C), 126.9, 126.8, 125.5, 112.6, 48.4, 47.1, 39.1; ATR-FTIR $\nu$ 3067, 3024, $1596,1567,1494,1471,1367,1230,1023 \mathrm{~cm}^{-1}$; HRMS (ESI) $\mathrm{m} / z$ calcd for $\mathrm{C}_{24} \mathrm{H}_{20} \mathrm{BrO}_{2}[\mathrm{M}+\mathrm{H}]^{+}$419.0641, found 419.0630 .

(2Z)-2-[Hydroxy(phenyl)methylidene]-4-(4-methylphenyl)-3phenylcyclopentan-1-one (2g). Purified in 98:2 to $97: 3$ petroleum ether/EtOAc; $80 \mathrm{mg}$ yield as a thick yellow oil (90\%); 1:7 keto:enol. Enol: ${ }^{1} \mathrm{H}$ NMR $\left(400 \mathrm{MHz}, \mathrm{CDCl}_{3}\right) \delta 7.57-7.54(\mathrm{~m}, 2 \mathrm{H}), 7.40-7.35$ (m, $1 \mathrm{H}), 7.29-7.26(\mathrm{~m}, 2 \mathrm{H}), 7.14-7.11(\mathrm{~m}, 3 \mathrm{H}), 6.93-6.91(\mathrm{~m}$, $2 \mathrm{H}), 6.85-6.78(\mathrm{~m}, 2 \mathrm{H}), 6.67-6.65(\mathrm{~m}, 2 \mathrm{H}), 4.36(\mathrm{~d}, J=7.4 \mathrm{~Hz}$, $1 \mathrm{H}), 3.84\left(\mathrm{dt}, J=14.1 \mathrm{~Hz}, J^{\prime}=7.2 \mathrm{~Hz}, 1 \mathrm{H}\right), 3.08\left(\mathrm{dd}, J=17.3 \mathrm{~Hz}, J^{\prime}\right.$ $=13.6 \mathrm{~Hz}, 1 \mathrm{H}), 2.61\left(\mathrm{dd}, J=17.3 \mathrm{~Hz}, J^{\prime}=7.1 \mathrm{~Hz}, 1 \mathrm{H}\right), 2.27(\mathrm{~s}, 3 \mathrm{H})$; ${ }^{13} \mathrm{C}\left\{{ }^{1} \mathrm{H}\right\}$ NMR $\left(101 \mathrm{MHz}, \mathrm{CDCl}_{3}\right) \delta 209.5,170.7,140.6,136.3$, 135.8, 134.0, 131.2, 128.7 (2C), 128.6 (2C), 128.4 (2C), 128.3 (2C), 128.2 (2C), 128.1 (2C), 126.7, 113.4, 51.9, 48.1, 40.1, 21.1. Keto: ${ }^{1} \mathrm{H}$ NMR $\left(400 \mathrm{MHz}, \mathrm{CDCl}_{3}\right) \delta 8.05-8.00(\mathrm{~m}, 2 \mathrm{H}), 7.52-7.48(\mathrm{~m}, 2 \mathrm{H})$, 7.30-7.26 (m, 2H), 7.15-7.08 (m, 3H), 7.00-6.96 (m, 2H), 6.84$6.78(\mathrm{~m}, 1 \mathrm{H}), 6.72(\mathrm{~d}, J=8.1 \mathrm{~Hz}, 2 \mathrm{H}), 4.86(\mathrm{~d}, J=8.6 \mathrm{~Hz}, 1 \mathrm{H}), 4.51$ $\left(\mathrm{dd}, J=8.6 \mathrm{~Hz}, J^{\prime}=6.8 \mathrm{~Hz}, 1 \mathrm{H}\right), 4.05(\mathrm{q}, J=6.9 \mathrm{~Hz}, 1 \mathrm{H}), 2.96(\mathrm{~d}, J=$ $6.9 \mathrm{~Hz}, 1 \mathrm{H}), 2.27(\mathrm{~s}, 3 \mathrm{H}) ;{ }^{13} \mathrm{C}\left\{{ }^{1} \mathrm{H}\right\}$ NMR $\left(101 \mathrm{MHz}, \mathrm{CDCl}_{3}\right) \delta$ 212.5, 194.6, 172.1, 142.2, 138.7, 136.4, 134.0, 133.7, 129.6, 128.9 (2C), 128.7 (2C), 128.6 (2C), 128.2 (2C), 128.0 (2C), 127.9 (2C), 61.1, 51.9, 45.1, 22.0; ATR-FTIR $\nu$ 3024, 1744, 1608, 1592, 1571, $1515,1492,1448,1365,1261,1227,1150,1098,1078,1030 \mathrm{~cm}^{-1}$; HRMS (ESI) $m / z$ calcd for $\mathrm{C}_{25} \mathrm{H}_{23} \mathrm{O}_{2}[\mathrm{M}+\mathrm{H}]^{+} 355.1693$, found 355.1677.

(2Z)-2-[Hydroxy(phenyl)methylidene]-3,4-bis(4-methylphenyl)cyclopentan-1-one (2h). Purified in 98:2 petroleum ether/EtOAc; 70 mg yield as a thick yellow oil (76\%); 1:5 keto:enol. Enol: ${ }^{1} \mathrm{H}$ NMR $\left(400 \mathrm{MHz}, \mathrm{CDCl}_{3}\right) \delta 7.58-7.51(\mathrm{~m}, 2 \mathrm{H}), 7.40-7.33(\mathrm{~m}, 1 \mathrm{H}), 7.28-$ $7.25(\mathrm{~m}, 2 \mathrm{H}), 6.91(\mathrm{~d}, J=7.8 \mathrm{~Hz}, 4 \mathrm{H}), 6.71-6.61(\mathrm{~m}, 4 \mathrm{H}), 4.29(\mathrm{~d}, J$ $=7.2 \mathrm{~Hz}, 1 \mathrm{H}), 3.79\left(\mathrm{dt}, J=14.0 \mathrm{~Hz}, J^{\prime}=7.1 \mathrm{~Hz}, 1 \mathrm{H}\right), 3.04(\mathrm{dd}, J=$ $\left.17.3 \mathrm{~Hz}, J^{\prime}=13.7 \mathrm{~Hz}, 1 \mathrm{H}\right), 2.57\left(\mathrm{dd}, J=17.3 \mathrm{~Hz}, J^{\prime}=7.0 \mathrm{~Hz}, 1 \mathrm{H}\right)$, $2.26(\mathrm{~s}, 6 \mathrm{H}) ;{ }^{13} \mathrm{C}\left\{{ }^{1} \mathrm{H}\right\}$ NMR $\left(101 \mathrm{MHz}, \mathrm{CDCl}_{3}\right) \delta 209.7,170.4$, 137.4, 136.3 (2C), 136.2, 136.0, 131.2, 129.0 (2C), 128.6 (2C), 128.6 (2C), 128.5 (2C), 128.3 (2C), 128.2 (2C), 113.8, 51.5, 48.2, 40.1, 21.2 (2C). Keto: ${ }^{1} \mathrm{H}$ NMR $\left(400 \mathrm{MHz}, \mathrm{CDCl}_{3}\right) \delta 8.02-7.96(\mathrm{~m}, 2 \mathrm{H})$, 7.59-7.55 (m, 1H), 7.51-7.42 (m, 3H), 7.29-7.24 (m, 2H), $6.97(\mathrm{~d}$, $J=7.8 \mathrm{~Hz}, 2 \mathrm{H}), 6.74-6.68(\mathrm{~m}, 3 \mathrm{H}), 4.79(\mathrm{~d}, J=8.5 \mathrm{~Hz}, 1 \mathrm{H}), 4.43$ $\left(\mathrm{dd}, J=8.5 \mathrm{~Hz}, J^{\prime}=6.8 \mathrm{~Hz}, 1 \mathrm{H}\right), 4.00(\mathrm{q}, J=6.9 \mathrm{~Hz}, 1 \mathrm{H}), 2.93(\mathrm{~d}, J=$ $6.9 \mathrm{~Hz}, 2 \mathrm{H}), 2.26(\mathrm{~s}, 6 \mathrm{H}) ;{ }^{13} \mathrm{C}\left\{{ }^{1} \mathrm{H}\right\}$ NMR $\left(101 \mathrm{MHz}, \mathrm{CDCl}_{3}\right) \delta$ $212.8,194.8,136.7,136.6,136.5,136.4,135.6,133.7,129.6$ (2C), 128.9 (4C), 128.7 (2C), 128.1 (4C), 61.4, 50.8, 45.2, 45.1, 21.1 (2C); ATR-FTIR $\nu$ 3020, 2913, 1744, 1573, 1513, 1494, 1361, $1223 \mathrm{~cm}^{-1}$; HRMS (ESI) $m / z$ calcd for $\mathrm{C}_{26} \mathrm{H}_{25} \mathrm{O}_{2}[\mathrm{M}+\mathrm{H}]^{+} 369.1849$, found 369.1862.

(2Z)-2-[Hydroxy(phenyl)methylidene]-4-(4-methoxyphenyl)-3phenylcyclopentan-1-one (2i). Purified in 98:2 petroleum ether/ EtOAc; $46 \mathrm{mg}$ yield as a thick yellow oil (50\%); 1:5 keto:enol. Enol: ${ }^{1} \mathrm{H}$ NMR $\left(400 \mathrm{MHz}, \mathrm{CDCl}_{3}\right) \delta 7.61-7.51(\mathrm{~m}, 2 \mathrm{H}), 7.39-7.33(\mathrm{~m}$, $1 \mathrm{H}), 7.28-7.24(\mathrm{~m}, 2 \mathrm{H}), 7.15-7.06(\mathrm{~m}, 3 \mathrm{H}), 6.82-6.75(\mathrm{~m}, 2 \mathrm{H})$, $6.65-6.63(\mathrm{~m}, 3 \mathrm{H}), 4.32(\mathrm{~d}, J=7.4 \mathrm{~Hz}, 1 \mathrm{H}), 3.82\left(\mathrm{dt}, J=14.0 \mathrm{~Hz}, J^{\prime}\right.$ $=7.2 \mathrm{~Hz}, 1 \mathrm{H}), 3.73(\mathrm{~s}, 3 \mathrm{H}), 3.02\left(\mathrm{dd}, J=17.3 \mathrm{~Hz}, J^{\prime}=13.6 \mathrm{~Hz}, 1 \mathrm{H}\right)$, $2.59\left(\mathrm{dd}, J=17.3 \mathrm{~Hz}, J^{\prime}=7.1 \mathrm{~Hz}, 1 \mathrm{H}\right) ;{ }^{13} \mathrm{C}\left\{{ }^{1} \mathrm{H}\right\}$ NMR $(101 \mathrm{MHz}$, $\left.\mathrm{CDCl}_{3}\right) \delta 209.5,170.7,158.4,140.7,134.0,131.2,129.6,129.2(2 \mathrm{C})$, 128.7 (2C), 128.4 (2C), 128.3 (4C), 126.7, 113.6, 113.4 (2C), 55.3, 52.0, 47.7, 40.2. Keto: ${ }^{1} \mathrm{H}$ NMR $\left(400 \mathrm{MHz}^{\mathrm{CDCl}}{ }_{3}\right) \delta 8.04-7.96(\mathrm{~m}$, $2 \mathrm{H}), 7.61-7.56(\mathrm{~m}, 1 \mathrm{H}), 7.50-7.45(\mathrm{~m}, 2 \mathrm{H}), 7.14-7.09(\mathrm{~m}, 3 \mathrm{H})$, $6.82-6.75(\mathrm{~m}, 2 \mathrm{H}), 6.70(\mathrm{~d}, J=2.8 \mathrm{~Hz}, 2 \mathrm{H}), 6.64(\mathrm{~d}, J=3.2 \mathrm{~Hz}$, $2 \mathrm{H}), 4.81(\mathrm{~d}, J=8.5 \mathrm{~Hz}, 0 \mathrm{H}), 4.46(\mathrm{dd}, J=8.4,6.8 \mathrm{~Hz}, 1 \mathrm{H}), 4.02(\mathrm{q}$, $J=6.9 \mathrm{~Hz}, 1 \mathrm{H}), 3.75(\mathrm{~s}, 2 \mathrm{H}), 2.96-2.90(\mathrm{~m}, 1 \mathrm{H}) ;{ }^{13} \mathrm{C}\left\{{ }^{1} \mathrm{H}\right\} \mathrm{NMR}$ $\left(101 \mathrm{MHz}, \mathrm{CDCl}_{3}\right) \delta 212.6,195.0,138.8,136.6,133.8,131.6,131.0$ (2C), 129.6, 129.1 (2C), 128.8 (2C), 128.3 (4C), 127.0, 113.4 (2C), 61.2, 56.3, 51.2, 45.1, 44.8; ATR-FTIR $\nu$ 2840, 1744, 1610, 1515,
$1365,1248,1180,1032 \mathrm{~cm}^{-1}$; HRMS (ESI) $m / z$ calcd for $\mathrm{C}_{25} \mathrm{H}_{23} \mathrm{O}_{3}$ $[\mathrm{M}+\mathrm{H}]^{+} 371.1642$, found 371.1638 .

Characterization of Demethylated OTHO (1i'). Methyl 4-(4Hydroxyphenyl)-6-oxo-3,4-diphenylhexanoate (1i'). Purified in 3:1 pentane/EtAOc; $25 \mathrm{mg}$ yield $(25 \%)$ as a white solid; $\mathrm{mp} 180-182{ }^{\circ} \mathrm{C}$ (from $\mathrm{MeOH}) ;{ }^{1} \mathrm{H}$ NMR $\left(400 \mathrm{MHz}, \mathrm{CDCl}_{3}\right) \delta 7.64(\mathrm{~d}, J=7.6 \mathrm{~Hz}$, $2 \mathrm{H}), 7.45(\mathrm{t}, J=7.3 \mathrm{~Hz}, 1 \mathrm{H}), 7.34-7.27(\mathrm{~m}, 6 \mathrm{H}), 7.21-7.12(\mathrm{~m}$, $3 \mathrm{H}), 6.72(\mathrm{~d}, J=7.7 \mathrm{~Hz}, 2 \mathrm{H}), 3.55\left(\mathrm{td}, J=11.3 \mathrm{~Hz}, J^{\prime}=10.2 \mathrm{~Hz}, J^{\prime \prime}=\right.$ $6.6 \mathrm{~Hz}, 1 \mathrm{H}), 3.47-3.21(\mathrm{~m}, 5 \mathrm{H}), 2.93\left(\mathrm{dd}, J=16.6 \mathrm{~Hz}, J^{\prime}=3.2 \mathrm{~Hz}\right.$, $1 \mathrm{H}), 2.67-2.30(\mathrm{~m}, 2 \mathrm{H}) ;{ }^{13} \mathrm{C}\left\{{ }^{1} \mathrm{H}\right\}$ NMR $\left(101 \mathrm{MHz}, \mathrm{CDCl}_{3}\right) \delta 199.3$, 173.2, 155.0, 142.6, 137.1, 133.8, 133.0, 129.4 (2C), 128.8 (2C), 128.5 (2C), 128.4 (2C), 128.0 (2C), 127.0, 115.8 (2C), 51.7, 47.3 (2C), 44.0, 40.3; ATR-FTIR $\nu$ 2204, 2160, 2190, 1726, 1680, 1514, 1231, $1153 \mathrm{~cm}^{-1}$; HRMS (ESI) $\mathrm{m} / z$ calcd for $\mathrm{C}_{25} \mathrm{H}_{25} \mathrm{O}_{4}[\mathrm{M}+\mathrm{H}]^{+}$ 389.1747, found 389.1748 .

Methyl 4-\{(3Z)-3-[Hydroxy(phenyl)methylidene]-4-oxo-2phenylcyclopentyl\}benzoate (2j). Purified in 94:6 to 93:7 petroleum ether/EtOAc; $50 \mathrm{mg}$ yield as a thick yellow oil $(50 \%) ; 1: 8$ keto:enol 1:8. Enol: ${ }^{1} \mathrm{H}$ NMR (400 MHz, $\left.\mathrm{CDCl}_{3}\right) \delta 7.79-7.73(\mathrm{~m}, 2 \mathrm{H}), 7.53-$ $7.50(\mathrm{~m}, 2 \mathrm{H}), 7.39-7.35(\mathrm{~m}, 1 \mathrm{H}), 7.28-7.24(\mathrm{~s}, 3 \mathrm{H}), 7.13-7.05(\mathrm{~m}$, $3 \mathrm{H}), 6.87-6.81(\mathrm{~m}, 2 \mathrm{H}), 6.78-6.75(\mathrm{~m}, 2 \mathrm{H}), 4.39(\mathrm{~d}, J=7.4 \mathrm{~Hz}$, $1 \mathrm{H}), 3.96-3.86(\mathrm{~m}, 4 \mathrm{H}), 3.11\left(\mathrm{dd}, J=17.3 \mathrm{~Hz}, J^{\prime}=13.4 \mathrm{~Hz}, 1 \mathrm{H}\right)$, $2.64\left(\mathrm{dd}, J=17.4 \mathrm{~Hz}, J^{\prime}=7.1 \mathrm{~Hz}, 1 \mathrm{H}\right) ;{ }^{13} \mathrm{C}\left\{{ }^{1} \mathrm{H}\right\}$ NMR $(101 \mathrm{MHz}$, $\left.\mathrm{CDCl}_{3}\right) \delta 208.6,171.3,167.0,144.6,140.2,133.9,131.4,129.3(2 \mathrm{C})$, 128.5 (4C), 128.4 (5C), 128.2 (2C), 127.0, 113.0, 52.1, 51.9, 48.3, 39.6. Keto: ${ }^{1} \mathrm{H}$ NMR $\left(400 \mathrm{MHz}, \mathrm{CDCl}_{3}\right) \delta 8.02\left(\mathrm{dt}, J=9.0 \mathrm{~Hz}, J^{\prime}=\right.$ $1.5 \mathrm{~Hz}, 2 \mathrm{H}), 8.00-7.94(\mathrm{~m}, 2 \mathrm{H}), 7.82-7.79(\mathrm{~m}, 2 \mathrm{H}), 7.62-7.57(\mathrm{~m}$, $1 \mathrm{H}), 7.51-7.47(\mathrm{~m}, 2 \mathrm{H}), 7.13-7.03(\mathrm{~m}, 2 \mathrm{H}), 6.94-6.86(\mathrm{~m}, 2 \mathrm{H})$, $6.78-6.75(\mathrm{~m}, 2 \mathrm{H}), 4.83(\mathrm{~d}, J=7.5 \mathrm{~Hz}, 1 \mathrm{H}), 4.51(\mathrm{t}, J=7.3 \mathrm{~Hz}, 1 \mathrm{H})$, $4.16(\mathrm{q}, J=7.3 \mathrm{~Hz}, 1 \mathrm{H}), 3.87(\mathrm{~s}, 3 \mathrm{H}), 3.05-2.92(\mathrm{~m}, 2 \mathrm{H}) ;{ }^{13} \mathrm{C}\left\{{ }^{1} \mathrm{H}\right\}$ NMR (101 MHz, $\left.\mathrm{CDCl}_{3}\right) \delta 211.6,194.2,145.0,138.3,136.3,130.0$, 129.7 (2C), 129.5 (2C), 128.8 (2C), 128.6 (3C), 128.1 (4C), 127.3, 61.6, 51.0, 45.6, 44.1, 35.3, 31.0; ATR-FTIR $\nu$ 3062, 2951, 1717, $1677,1607,1570,1493,1367,1277,1097,1019 \mathrm{~cm}^{-1}$; HRMS (ESI) $m / z$ calcd for $\mathrm{C}_{26} \mathrm{H}_{23} \mathrm{O}_{4}[\mathrm{M}+\mathrm{H}]^{+}$399.1591, found 399.1607.

(2Z)-3-(4-Chlorophenyl)-2-[hydroxy(phenyl)methylidene]-4-phenylcyclopentan-1-one (2k). Purified in 99:1 to 95:5 petroleum ether/ EtOAc; $55 \mathrm{mg}$ yield as a thick transparent oil (59\%); 1:4 keto:enol. Enol: ${ }^{1} \mathrm{H}$ NMR (400 MHz, $\left.\mathrm{CDCl}_{3}\right) \delta$ 7.52-7.44 (m, 2H), 7.42-7.35 (m, 1H), 7.30-7.26 (m, 1H), 7.22-7.16 (m, 1H), 7.16-7.09 (m, $3 \mathrm{H}), 7.09-7.02(\mathrm{~m}, 2 \mathrm{H}), 6.85-6.74(\mathrm{~m}, 2 \mathrm{H}), 6.74-6.64(\mathrm{~m}, 2 \mathrm{H})$, $4.35(\mathrm{~d}, J=7.4 \mathrm{~Hz}, 1 \mathrm{H}), 3.93-3.82(\mathrm{~m}, 1 \mathrm{H}), 3.09-2.94(\mathrm{~m}, 1 \mathrm{H})$, 2.67-2.57 (m, $1 \mathrm{H}) ;{ }^{13} \mathrm{C}\left\{{ }^{1} \mathrm{H}\right\}$ NMR $\left(101 \mathrm{MHz}, \mathrm{CDCl}_{3}\right) \delta$ 212.1, $171.7,139.5,138.9,131.8,130.2$ (2C), 130.0, 128.8 (2C), 128.7 (2C), 128.6 (2C), 128.5 (4C), 128.4, 127.4, 113.2, 51.7, 48.5, 39.9. Keto: ${ }^{1} \mathrm{H}$ NMR $\left(400 \mathrm{MHz}, \mathrm{CDCl}_{3}\right) \delta 8.01-7.96(\mathrm{~m}, 2 \mathrm{H}), 7.62-7.56$ $(\mathrm{m}, 1 \mathrm{H}), 7.52-7.44(\mathrm{~m}, 1 \mathrm{H}), 7.30-7.26(\mathrm{~m}, 1 \mathrm{H}), 7.22-7.16(\mathrm{~m}$, $2 \mathrm{H}), 7.16-7.09(\mathrm{~m}, 3 \mathrm{H}), 6.85-6.74(\mathrm{~m}, 2 \mathrm{H}), 6.74-6.64(\mathrm{~m}, 2 \mathrm{H})$, $4.76(\mathrm{~d}, J=9.1 \mathrm{~Hz}, 1 \mathrm{H}), 4.56-4.47(\mathrm{~m}, 1 \mathrm{H}), 4.06-3.99(\mathrm{q}, J=6.7$ $\mathrm{Hz}, 1 \mathrm{H}), 3.09-2.94(\mathrm{~m}, 1 \mathrm{H}) ;{ }^{13} \mathrm{C}\left\{{ }^{1} \mathrm{H}\right\}$ NMR $\left(101 \mathrm{MHz}, \mathrm{CDCl}_{3}\right) \delta$ 212.1, 194.5, 139.7, 137.5, 134.2, 134.2, 132.9, 130.0, 129.8, 129.1, $128.8,128.8$ (2C), 128.7(2C), 128.6 (2C), 128.4 (2C), 127.5, 61.3, 50.7, 45.6, 45.4; ATR-FTIR $\nu$ 2956, 2915, 1546, 1566, 1489, 1351, 1257, 1227, 1088, $1014 \mathrm{~cm}^{-1}$; HRMS (ESI) $\mathrm{m} / z$ calcd for $\mathrm{C}_{24} \mathrm{H}_{20} \mathrm{ClO}_{2}[\mathrm{M}+\mathrm{H}]^{+}$375.1146, found 375.1145.

(2Z)-3-(4-Bromophenyl)-2-[hydroxy(phenyl)methylidene]-4-phenylcyclopentan-1-one (2l). Purified in 99:1 to 97:3 petroleum ether/ EtOAc; $75 \mathrm{mg}$ yield as a thick yellow oil (72\%); 1:10 keto:enol. Enol: ${ }^{1} \mathrm{H}$ NMR $\left(400 \mathrm{MHz}, \mathrm{CDCl}_{3}\right) \delta 7.54-7.46(\mathrm{~m}, 2 \mathrm{H}), 7.44-7.36(\mathrm{~m}$, $1 \mathrm{H}), 7.29-7.25(\mathrm{~m}, 2 \mathrm{H}), 7.24-7.19(\mathrm{~m}, 2 \mathrm{H}), 7.16-7.10(\mathrm{~m}, 3 \mathrm{H})$, 6.82-6.74 (m, 2H), 6.69-6.58 (m, $2 \mathrm{H}), 4.34(\mathrm{~d}, J=7.4 \mathrm{~Hz}, 1 \mathrm{H})$, $3.94-3.81(\mathrm{~m}, 1 \mathrm{H}), 3.04\left(\mathrm{dd}, J=17.4 \mathrm{~Hz}, J^{\prime}=13.5 \mathrm{~Hz}, 1 \mathrm{H}\right), 2.63$ $\left(\mathrm{dd}, J=17.4 \mathrm{~Hz}, J^{\prime}=7.1 \mathrm{~Hz}, 1 \mathrm{H}\right) ;{ }^{13} \mathrm{C}\left\{{ }^{1} \mathrm{H}\right\}$ NMR $(101 \mathrm{MHz}$, $\left.\mathrm{CDCl}_{3}\right) \delta 208.9,171.3,139.6,138.5,133.8,131.4,131.3(2 \mathrm{C}), 130.2$ (2C), 128.4 (2C), 128.3 (2C), 128.2 (2C), 128.1 (2C), 127.1, 120.7, 112.7, 51.4, 48.1, 39.5. Keto: ${ }^{1} \mathrm{H}$ NMR $\left(400 \mathrm{MHz}, \mathrm{CDCl}_{3}\right) \delta 8.04-$ $7.97(\mathrm{~m}, 2 \mathrm{H}), 7.64-7.56(\mathrm{~m}, 1 \mathrm{H}), 7.53-7.45(\mathrm{~m}, 1 \mathrm{H}), 7.30-7.24$ $(\mathrm{m}, 1 \mathrm{H}), 7.24-7.18(\mathrm{~m}, 2 \mathrm{H}), 7.16-7.11(\mathrm{~m}, 3 \mathrm{H}), 6.84-6.79(\mathrm{~s}, 2 \mathrm{H})$, $6.68-6.62(\mathrm{~m}, 2 \mathrm{H}), 4.77(\mathrm{~d}, J=9.2 \mathrm{~Hz}, 1 \mathrm{H}), 4.51\left(\mathrm{dd}, J=9.2 \mathrm{~Hz}, J^{\prime}\right.$ $=6.9 \mathrm{~Hz}, 1 \mathrm{H}), 4.03(\mathrm{q}, J=6.7 \mathrm{~Hz}, 1 \mathrm{H}), 2.97(\mathrm{~d}, J=6.6 \mathrm{~Hz}, 1 \mathrm{H})$; 
${ }^{13} \mathrm{C}\left\{{ }^{1} \mathrm{H}\right\}$ NMR $\left(101 \mathrm{MHz}, \mathrm{CDCl}_{3}\right) \delta 211.6,194.1,139.3,137.7$, 136.5, 133.9, 131.3, 129.8 (2C), 129.6 (2C), 128.8 (2C), 128.4 (2C), 128.1 (2C), 127.1 (2C), 121.0, 60.8, 50.4, 45.2, 45.0; ATR-FTIR $\nu$ 2959, 1742, 1644, 1592, 1565, 1486, 1351, $1230 \mathrm{~cm}^{-1}$; HRMS (ESI) $m / z$ calcd for $\mathrm{C}_{24} \mathrm{H}_{20} \mathrm{BrO}_{2}[\mathrm{M}+\mathrm{H}]^{+} 419.0641$, found 419.0641 .

(2Z)-2-[Hydroxy(phenyl)methylidene]-3-(4-methylphenyl)-4phenylcyclopentan-1-one (2m). Purified in 98:2 to 97:3 petroleum ether/EtOAc; $62 \mathrm{mg}$ yield as a thick yellow oil (70\%); 1:10 keto:enol. Enol: ${ }^{1} \mathrm{H}$ NMR $\left(400 \mathrm{MHz}, \mathrm{CDCl}_{3}\right) \delta 7.61-7.54(\mathrm{~m}, 2 \mathrm{H}), 7.43-7.34$ $(\mathrm{m}, 1 \mathrm{H}), 7.30-7.26(\mathrm{~s}, 2 \mathrm{H}), 7.16-7.07(\mathrm{~m}, 3 \mathrm{H}), 6.95-6.89(\mathrm{~m}, 2 \mathrm{H})$, 6.84-6.76 (m, 2H), 6.71-6.64 (m, 2H), $4.35(\mathrm{~d}, J=7.3 \mathrm{~Hz}, 1 \mathrm{H})$, $3.85\left(\mathrm{dt}, J=14.0 \mathrm{~Hz}, J^{\prime}=7.1 \mathrm{~Hz}, 1 \mathrm{H}\right), 3.10\left(\mathrm{dd}, J=17.3 \mathrm{~Hz}, J^{\prime}=13.7\right.$ $\mathrm{Hz}, 1 \mathrm{H}), 2.62\left(\mathrm{dd}, J=17.2 \mathrm{~Hz}, J^{\prime}=7.0 \mathrm{~Hz}, 1 \mathrm{H}\right), 2.26(\mathrm{~s}, 3 \mathrm{H})$; ${ }^{13} \mathrm{C}\left\{{ }^{1} \mathrm{H}\right\}$ NMR $\left(101 \mathrm{MHz}, \mathrm{CDCl}_{3}\right) \delta 209.5,170.5,139.0,137.3$, 136.2, 134.0, 131.2, 128.9 (2C), 128.5 (2C), 128.4 (2C), 128.3 (2C), 128.2 (2C), 127.9 (2C), 126.7, 113.6, 51.6, 48.4, 39.8, 21.1. Keto: ${ }^{1} \mathrm{H}$ NMR (400 MHz, $\left.\mathrm{CDCl}_{3}\right) \delta 8.07-7.99(\mathrm{~m}, 2 \mathrm{H}), 7.52-7.45(\mathrm{~m}, 2 \mathrm{H})$, $7.30-7.26(\mathrm{~s}, 2 \mathrm{H}), 7.20-7.17(\mathrm{~m}, 3 \mathrm{H}), 6.95-6.89(\mathrm{~m}, 1 \mathrm{H}), 6.88-$ $6.82(\mathrm{~m}, 2 \mathrm{H}), 6.72-6.62(\mathrm{~m}, 2 \mathrm{H}), 4.83(\mathrm{~d}, J=8.3 \mathrm{~Hz}, 1 \mathrm{H}), 4.48(\mathrm{dd}$, $\left.J=8.3 \mathrm{~Hz}, J^{\prime}=6.9 \mathrm{~Hz}, 1 \mathrm{H}\right), 4.07(\mathrm{q}, J=7.0 \mathrm{~Hz}, 1 \mathrm{H}), 3.02-2.91(\mathrm{~m}$, $2 \mathrm{H}), 2.24(\mathrm{~s}, 3 \mathrm{H}) ;{ }^{13} \mathrm{C}\left\{{ }^{1} \mathrm{H}\right\}$ NMR $\left(101 \mathrm{MHz}, \mathrm{CDCl}_{3}\right) \delta 212.5,194.7$, $139.7,136.6,136.5,135.5,133.7,129.6$ (2C), 128.9 (2C), 128.7 (2C), 128.2 (2C), 128.1 (2C), 128.0 (2C), 126.8, 61.4, 50.8, 45.5, 44.8, 21.1; ATR-FTIR $\nu$ 2917, 1740, 1606, 1569, 1494, 1369, 1265 $\mathrm{cm}^{-1}$; HRMS (ESI) $\mathrm{m} / z$ calcd for $\mathrm{C}_{25} \mathrm{H}_{23} \mathrm{O}_{2}[\mathrm{M}+\mathrm{H}]^{+}$355.1693, found 355.1701 .

4-\{(2Z)-2-[Hydroxy (phenyl)methylidene]-3-methylidene-5phenylcyclopentyl\}benzonitrile (2n). Purified in 9:1 to 4:1 pentane/ EtOAc; $72 \mathrm{mg}$ yield as a thick pink oil (79\%); >1:7 keto:enol. Enol: ${ }^{1} \mathrm{H} \mathrm{NMR}\left(400 \mathrm{MHz}, \mathrm{CDCl}_{3}\right) \delta 7.48-7.41(\mathrm{~m}, 2 \mathrm{H}), 7.36(\mathrm{dd}, J=9.5$ $\left.\mathrm{Hz}, J^{\prime}=7.8 \mathrm{~Hz}, 3 \mathrm{H}\right), 7.28-7.24(\mathrm{~m}, 2 \mathrm{H}), 7.12\left(\mathrm{dt}, J=5.4 \mathrm{~Hz}, J^{\prime}=2.7\right.$ $\mathrm{Hz}, 3 \mathrm{H}), 6.87(\mathrm{~d}, J=8.1 \mathrm{~Hz}, 2 \mathrm{H}), 6.76\left(\mathrm{dd}, J=7.1 \mathrm{~Hz}, J^{\prime}=2.4 \mathrm{~Hz}\right.$, $2 \mathrm{H}), 4.48(\mathrm{~d}, J=7.6 \mathrm{~Hz}, 1 \mathrm{H}), 3.95\left(\mathrm{dt}, J=12.9 \mathrm{~Hz}, J^{\prime}=7.4 \mathrm{~Hz}, 1 \mathrm{H}\right)$, $3.03\left(\mathrm{dd}, J=17.5 \mathrm{~Hz}, J^{\prime}=13.0 \mathrm{~Hz}, 1 \mathrm{H}\right), 2.69\left(\mathrm{dd}, J=17.5 \mathrm{~Hz}, J^{\prime}=\right.$ $7.2 \mathrm{~Hz}, 1 \mathrm{H}) ;{ }^{13} \mathrm{C}\left\{{ }^{1} \mathrm{H}\right\}$ NMR $\left(101 \mathrm{MHz}, \mathrm{CDCl}_{3}\right) \delta 208.2,172.2$, $146.4,138.1,133.6,131.9$ (2C), 131.6, 129.1 (2C), 128.6, 128.4 (2C), 128.3 (2C), 128.1 (2C), 127.9 (2C), 127.3, 111.9, 110.6, 52.0, 47.9, 39.4. Keto: ${ }^{1} \mathrm{H}$ NMR $\left(400 \mathrm{MHz}, \mathrm{CDCl}_{3}\right) \delta 8.06-7.97(\mathrm{~m}, 2 \mathrm{H})$, $7.61(\mathrm{t}, J=7.4 \mathrm{~Hz}, 1 \mathrm{H}), 7.50(\mathrm{t}, J=7.7 \mathrm{~Hz}, 2 \mathrm{H}), 7.21-7.17(\mathrm{~m}, 3 \mathrm{H})$, $6.89-6.86(\mathrm{~m}, 3 \mathrm{H}), 6.81-6.78(\mathrm{~m}, 3 \mathrm{H}), 4.82(\mathrm{~d}, J=9.6 \mathrm{~Hz}, 1 \mathrm{H})$, $4.64\left(\mathrm{dd}, J=9.6 \mathrm{~Hz}, J^{\prime}=6.9 \mathrm{~Hz}, 1 \mathrm{H}\right), 4.06\left(\mathrm{td}, J=7.1 \mathrm{~Hz}, J^{\prime}=5.1\right.$ $\mathrm{Hz}, 1 \mathrm{H}), 3.06-2.96(\mathrm{~m}, 2 \mathrm{H}) ;{ }^{13} \mathrm{C}\left\{{ }^{1} \mathrm{H}\right\}$ NMR $\left(101 \mathrm{MHz}, \mathrm{CDCl}_{3}\right) \delta$ 210.7, 193.5, 144.3, 139.0, 136.3, 134.0, 132.0 (2C), 129.7 (2C), 128.9, 128.8 (2C), 128.6 (2C), 128.4 (2C), 128.3 (2C), 127.4, 118.8, 110.9, 60.2, 50.7, 45.1; ATR-FTIR $\nu$ 3029, 2228, 1743, 1607, 1570, $1493,1361,1227,1151 \mathrm{~cm}^{-1}$; HRMS (ESI) $\mathrm{m} / z$ calcd for $\mathrm{C}_{25} \mathrm{H}_{20} \mathrm{NO}_{2}[\mathrm{M}+\mathrm{H}]^{+}$366.1489, found 366.1489.

(2Z)-2-[Hydroxy(4-methylphenyl)methylidene]-3,4-diphenylcyclopentan-1-one (20). Purified in 99:1 to 95:5 petroleum ether/ EtOAc; $52 \mathrm{mg}$ yield as a thick transparent oil (56\%); >1:20 keto:enol. Enol: ${ }^{1} \mathrm{H}$ NMR $\left(400 \mathrm{MHz}, \mathrm{CDCl}_{3}\right) \delta 7.51-7.45(\mathrm{~m}, 2 \mathrm{H}), 7.27-7.21$ $(\mathrm{m}, 2 \mathrm{H}), 7.17-7.04(\mathrm{~m}, 6 \mathrm{H}), 6.80-6.73(\mathrm{~m}, 4 \mathrm{H}), 4.33(\mathrm{~d}, J=7.4 \mathrm{~Hz}$, $1 \mathrm{H})$, 3.94-3.83 (m, $1 \mathrm{H}), 3.59-3.03(\mathrm{~m}, 1 \mathrm{H}), 2.68-2.59(\mathrm{~m}, 1 \mathrm{H})$; ${ }^{13} \mathrm{C}\left\{{ }^{1} \mathrm{H}\right\}$ NMR $\left(101 \mathrm{MHz}, \mathrm{CDCl}_{3}\right) \delta 212.6,212.3,209.7,193.5$, $169.9,140.7,140.6,139.8,139.1,138.8,137.8,135.2,132.7,131.4$, $130.1,129.4,129.0,128.9,128.7,128.5,128.3,127.4,127.2,113.4$, 77.2, 61.6, 52.3, 48.6, 45.8, 45.2, 40.1; ATR-FTIR $\nu$ 3027, 1591, 1487, $1360,1259,1090,1011 \mathrm{~cm}^{-1}$; HRMS (ESI) $\mathrm{m} / z$ calcd for $\mathrm{C}_{24} \mathrm{H}_{20} \mathrm{ClO}_{2}[\mathrm{M}+\mathrm{H}]^{+} 375.1139$, found 375.1146.

(2Z)-2-[(4-Bromophenyl)(hydroxy)methylidene]-3,4-diphenylcyclopentan-1-one (2p). Purified in $98: 2$ to $96: 4$ petroleum ether/ EtOAc; $79 \mathrm{mg}$ yield as a thick yellow oil (75\%); 1:18 keto:enol. Enol: ${ }^{1} \mathrm{H}$ NMR $\left(400 \mathrm{MHz}, \mathrm{CDCl}_{3}\right) \delta 7.42-7.38(\mathrm{~m}, 4 \mathrm{H}), 7.14-7.06(\mathrm{~m}$, $6 \mathrm{H}), 6.81-6.72(\mathrm{~m}, 4 \mathrm{H}), 4.33(\mathrm{~d}, J=7.4 \mathrm{~Hz}, 1 \mathrm{H}), 3.88(\mathrm{dt}, J=14.1$ $\left.\mathrm{Hz}, J^{\prime}=7.3 \mathrm{~Hz}, 1 \mathrm{H}\right), 3.10\left(\mathrm{dd}, J=17.4 \mathrm{~Hz}, J^{\prime}=13.6 \mathrm{~Hz}, 1 \mathrm{H}\right), 2.64$ $\left(\mathrm{dd}, J=17.4 \mathrm{~Hz}, J^{\prime}=7.1 \mathrm{~Hz}, 1 \mathrm{H}\right) ;{ }^{13} \mathrm{C}\left\{{ }^{1} \mathrm{H}\right\} \operatorname{NMR}(101 \mathrm{MHz}$, $\left.\mathrm{CDCl}_{3}\right) \delta 209.4,169.6,140.2,138.7,132.8,131.6$ (2C), 129.9 (2C), 128.5 (2C), $128.4(2 \mathrm{C}), 128.2(2 \mathrm{C}), 128.0(2 \mathrm{C}), 126.9,126.9,126.0$, 113.5, 51.9, 48.2, 39.8. Keto: ${ }^{1} \mathrm{H}$ NMR $\left(400 \mathrm{MHz}^{\mathrm{CDCl}}{ }_{3}\right) \delta 7.89(\mathrm{~d}$, $J=8.7 \mathrm{~Hz}, 2 \mathrm{H}), 7.62(\mathrm{~d}, J=8.5 \mathrm{~Hz}, 2 \mathrm{H}), 7.56-7.52(\mathrm{~m}, 1 \mathrm{H}), 7.35-$ $7.29(\mathrm{~m}, 1 \mathrm{H}), 7.18-7.07(\mathrm{~m}, 6 \mathrm{H}), 6.84-6.73(\mathrm{~m}, 2 \mathrm{H}), 4.77(\mathrm{~d}, J=$ $8.4 \mathrm{~Hz}, 1 \mathrm{H}), 4.52\left(\mathrm{dd}, J=8.6 \mathrm{~Hz}, J^{\prime}=6.9 \mathrm{~Hz}, 1 \mathrm{H}\right), 4.06(\mathrm{q}, J=7.0$ $\mathrm{Hz}, 1 \mathrm{H}), 3.01-2.94(\mathrm{~m}, 2 \mathrm{H}) ;{ }^{13} \mathrm{C}\left\{{ }^{1} \mathrm{H}\right\}$ NMR $\left(101 \mathrm{MHz}, \mathrm{CDCl}_{3}\right) \delta$ $211.9,193.3,139.4,138.5,135.3,132.1$ (2C), 131.2 (2C), 128.3 (2C), 128.2 (2C), 128.1 (4C), 127.1, 127.0, 126.0, 61.2, 50.9, 45.4, 44.8; ATR-FTIR $\nu 1588,1485,1359,1257,1234,1071,1007 \mathrm{~cm}^{-1}$; HRMS (ESI) $m / z$ calcd for $\mathrm{C}_{24} \mathrm{H}_{20} \mathrm{BrO}_{2}[\mathrm{M}+\mathrm{H}]^{+} 419.0641$, found 419.0638 .

(2Z)-2-[Hydroxy(4-methylphenyl)methylidene]-3,4-diphenylcyclopentan-1-one (2q). Purified in 99:1 to 95:5 petroleum ether/ EtOAc; $51 \mathrm{mg}$ yield as a thick transparent oil (58\%); 1:10 keto:enol. Enol: ${ }^{1} \mathrm{H}$ NMR $\left(400 \mathrm{MHz}, \mathrm{CDCl}_{3}\right) \delta 7.49-7.44(\mathrm{~m}, 2 \mathrm{H}), 7.14-7.05$ $(\mathrm{m}, 8 \mathrm{H}), 6.85-6.69(\mathrm{~m}, 4 \mathrm{H}), 4.38(\mathrm{~d}, J=7.4 \mathrm{~Hz}, 1 \mathrm{H}), 3.87(\mathrm{dt}, J=$ $\left.13.6 \mathrm{~Hz}, J^{\prime}=7.2 \mathrm{~Hz}, 1 \mathrm{H}\right), 3.09\left(\mathrm{dd}, J=17.3 \mathrm{~Hz}, J^{\prime}=13.6 \mathrm{~Hz}, 1 \mathrm{H}\right)$, $2.62\left(\mathrm{dd}, J=17.3 \mathrm{~Hz}, J^{\prime}=7.1 \mathrm{~Hz}, 1 \mathrm{H}\right), 2.32(\mathrm{~s}, 3 \mathrm{H}) ;{ }^{13} \mathrm{C}\left\{{ }^{1} \mathrm{H}\right\} \mathrm{NMR}$ $\left(101 \mathrm{MHz}, \mathrm{CDCl}_{3}\right) \delta 209.0,171.2,141.9,140.6,139.0,131.1,129.1$ (2C), 128.6 (2C), 128.5 (2C), 128.2 (4C), 128.0 (2C), 126.8, 126.7, 112.8, 52.1, 48.4, 39.7, 21.6. Keto: ${ }^{1} \mathrm{H}$ NMR (400 $\left.\mathrm{MHz}, \mathrm{CDCl}_{3}\right) \delta$ $7.93(\mathrm{~d}, J=8.3 \mathrm{~Hz}, 2 \mathrm{H}), 7.28\left(\mathrm{dt}, J=8.0 \mathrm{~Hz}, J^{\prime}=0.8 \mathrm{~Hz}, 3 \mathrm{H}\right), 7.18-$ $7.14(\mathrm{~m}, 3 \mathrm{H}), 7.11-7.04(\mathrm{~m}, 2 \mathrm{H}), 6.90-6.88(\mathrm{~m}, 2 \mathrm{H}), 6.83-6.81$ $(\mathrm{m}, 4 \mathrm{H}), 4.82(\mathrm{~d}, J=8.2 \mathrm{~Hz}, 1 \mathrm{H}), 4.51\left(\mathrm{dd}, J=8.2 \mathrm{~Hz}, J^{\prime}=7.0 \mathrm{~Hz}\right.$, $1 \mathrm{H}), 4.08(\mathrm{q}, J=7.0 \mathrm{~Hz}, 1 \mathrm{H}), 3.01-2.91(\mathrm{~m}, 1 \mathrm{H}), 2.42(\mathrm{~s}, 3 \mathrm{H})$; ${ }^{13} \mathrm{C}\left\{{ }^{1} \mathrm{H}\right\}$ NMR $\left(101 \mathrm{MHz}, \mathrm{CDCl}_{3}\right) \delta 212.5,194.0,144.8,139.7$, $138.8,134.1,129.8$ (2C), 129.5 (2C), 128.2 (6C), 128.1 (2C), 127.0, 126.9, 61.2, 51.1, 45.5, 44.7 21.8; ATR-FTIR $\nu$ 3022, 1744, 1632, $1595,1495,1364,1225,1149,1098 \mathrm{~cm}^{-1}$; HRMS (ESI) $\mathrm{m} / z$ calcd for $\mathrm{C}_{25} \mathrm{H}_{23} \mathrm{O}_{2}[\mathrm{M}+\mathrm{H}]^{+}$355.1693, found 355.1698.

Methyl 4-\{Hydroxy[(1Z)-5-oxo-2,3-diphenylcyclopentylidene]methyl\}benzoate (2r). Purified in 99:1 to $95: 5$ petroleum ether/ EtOAc; $41 \mathrm{mg}$ yield as a thick transparent oil (41\%); >1:20 keto:enol. Enol: ${ }^{1} \mathrm{H}$ NMR (400 MHz, $\left.\mathrm{CDCl}_{3}\right) \delta 8.06-7.77(\mathrm{~m}, 2 \mathrm{H}), 7.68-7.48$ (m, $2 \mathrm{H}), 7.16-7.00(\mathrm{~m}, 6 \mathrm{H}), 6.82-6.69(\mathrm{~m}, 4 \mathrm{H}), 4.34(\mathrm{~d}, J=7.4 \mathrm{~Hz}$, $1 \mathrm{H}), 3.88(\mathrm{~s}, 3 \mathrm{H}), 3.10\left(\mathrm{dd}, J=17.5 \mathrm{~Hz}, J^{\prime}=13.5 \mathrm{~Hz}, 1 \mathrm{H}\right), 2.65(\mathrm{dd}$, $\left.J=17.4 \mathrm{~Hz}, J^{\prime}=7.1 \mathrm{~Hz}, 1 \mathrm{H}\right) ;{ }^{13} \mathrm{C}\left\{{ }^{1} \mathrm{H}\right\} \operatorname{NMR}\left(101 \mathrm{MHz}, \mathrm{CDCl}_{3}\right) \delta$ 209.9, 169.2, 166.4, 140.3, 138.7, 138.0, 132.1, 129.5 (2C), 128.6 (2C), 128.4 (2C), 128.3 (2C), 128.2 (2C), 128.0 (2C), 126.9, 114.3, 77.2, 52.5, 51.9, 48.2, 39.9; ATR-FTIR $\nu$ 2957, 1712, 1636, 1593, $1426,1371,1283,1112,1018 \mathrm{~cm}^{-1}$; HRMS (ESI) $\mathrm{m} / z$ calcd for $\mathrm{C}_{26} \mathrm{H}_{23} \mathrm{O}_{4}[\mathrm{M}+\mathrm{H}]^{+}$399.1591, found 399.1597 .

4-\{Hydroxy[(1Z)-5-oxo-2,3-diphenylcyclopentylidene]methyl\}benzonitrile (2s). Purified in 9:1 to 4:1 pentane/EtOAc; $55 \mathrm{mg}$ yield as an orange solid (60\%); mp $122-125{ }^{\circ} \mathrm{C}$; >1:10 keto:enol. Enol: ${ }^{1} \mathrm{H}$ $\operatorname{NMR}\left(400 \mathrm{MHz}, \mathrm{CDCl}_{3}\right) \delta 7.61(\mathrm{~d}, J=8.5 \mathrm{~Hz}, 2 \mathrm{H}), 7.53(\mathrm{~d}, J=8.5$ $\mathrm{Hz}, 2 \mathrm{H}), 7.15-7.05(\mathrm{~m}, 6 \mathrm{H}), 6.75\left(\mathrm{td}, J=7.5 \mathrm{~Hz}, J^{\prime}=2.0 \mathrm{~Hz}, 4 \mathrm{H}\right)$, $4.32(\mathrm{~d}, J=7.4 \mathrm{~Hz}, 1 \mathrm{H}), 3.89\left(\mathrm{dt}, J=13.0 \mathrm{~Hz}, J^{\prime}=7.2 \mathrm{~Hz}, 1 \mathrm{H}\right), 3.10$ $\left(\mathrm{dd}, J=17.5 \mathrm{~Hz}, J^{\prime}=13.4 \mathrm{~Hz}, 1 \mathrm{H}\right), 2.67\left(\mathrm{dd}, J=17.5 \mathrm{~Hz}, J^{\prime}=7.1 \mathrm{~Hz}\right.$, $1 \mathrm{H}) ;{ }^{13} \mathrm{C}\left\{{ }^{1} \mathrm{H}\right\} \mathrm{NMR}\left(101 \mathrm{MHz}, \mathrm{CDCl}_{3}\right) \delta 210.1,167.7,139.9,138.4$, 138.1, 132.0 (2C), 128.8 (2C), 128.5 (2C), 128.4 (2C), 128.1 (4C), 127.1, 127.0, 114.7, $114.4(2 \mathrm{C}), 51.7,48.1,39.9$. Keto: ${ }^{1} \mathrm{H}$ NMR (400 $\left.\mathrm{MHz}, \mathrm{CDCl}_{3}\right) \delta 8.10(\mathrm{~d}, J=8.2 \mathrm{~Hz}, 2 \mathrm{H}), 7.78(\mathrm{~d}, J=8.2 \mathrm{~Hz}, 2 \mathrm{H})$, $7.18-7.13(\mathrm{~m}, 6 \mathrm{H}), 6.84-6.74(\mathrm{~m}, 4 \mathrm{H}), 4.79(\mathrm{~d}, J=8.9 \mathrm{~Hz}, 1 \mathrm{H})$, $4.53\left(\mathrm{dd}, J=8.9 \mathrm{~Hz}, J^{\prime}=6.9 \mathrm{~Hz}, 1 \mathrm{H}\right), 4.05(\mathrm{q}, J=6.8 \mathrm{~Hz}, 1 \mathrm{H}), 2.99$ $\left(\mathrm{dd}, J=6.7 \mathrm{~Hz}, J^{\prime}=4.6 \mathrm{~Hz}, 2 \mathrm{H}\right) ;{ }^{13} \mathrm{C}\left\{{ }^{1} \mathrm{H}\right\}$ NMR $\left(101 \mathrm{MHz}, \mathrm{CDCl}_{3}\right)$ $\delta$ 211.4, 193.1, 139.5, 139.2, 138.2, 132.5 (2C), 130.0 (2C), 128.3 (4C), 128.0 (4C), 127.2, 127.1, 116.9, 114.4, 61.5, 50.8, 45.4, 45.0; ATR-FTIR $\nu 3030,1637,1585,1495,1345,1261,1224,1148, \mathrm{~cm}^{-1}$; HRMS (ESI) $m / z$ calcd for $\mathrm{C}_{25} \mathrm{H}_{20} \mathrm{NO}_{2}[\mathrm{M}+\mathrm{H}]^{+} 366.1489$, found 366.1494 .

(Z)-2-[Hydroxy(phenyl)methylene]-3-(4-methoxyphenyl)-4-phenylcyclopentan-1-one (2t). Purified in 8:1 to 4:1 pentane/EtOAc; 43 mg yield as a yellow semisolid (46\%); >1:10 keto:enol. Enol: ${ }^{1} \mathrm{H}$ NMR $\left(400 \mathrm{MHz}, \mathrm{CDCl}_{3}\right) \delta 7.55-7.53(\mathrm{~m}, 2 \mathrm{H}), 7.41-7.33(\mathrm{~m}, 1 \mathrm{H})$, 7.31-7.22 (m, 2H), 7.14-7.05 (m, 3H), 6.79-6.76 (m, 2H), 6.72$6.59(\mathrm{~m}, 4 \mathrm{H}), 4.31(\mathrm{~d}, J=7.2 \mathrm{~Hz}, 1 \mathrm{H}), 3.89-3.78(\mathrm{~m}, 1 \mathrm{H}), 3.73(\mathrm{~s}$, $3 \mathrm{H}), 3.06\left(\mathrm{dd}, J=17.3 \mathrm{~Hz}, J^{\prime}=13.6 \mathrm{~Hz}, 1 \mathrm{H}\right), 2.60(\mathrm{dd}, J=17.3 \mathrm{~Hz}$, $\left.J^{\prime}=7.0 \mathrm{~Hz}, 1 \mathrm{H}\right) ;{ }^{13} \mathrm{C}\left\{{ }^{1} \mathrm{H}\right\}\left(101 \mathrm{MHz}, \mathrm{CDCl}_{3}\right) \delta 209.5,170.7,158.4$, 139.1, 132.6, 131.3, 129.6 (3C), 128.5 (2C), 128.4 (2C), 128.3 (2C), 128.0 (2C), 126.8, 113.7 (2C), 113.6, 55.3, 51.2, 48.4, 39.8. Keto: ${ }^{1} \mathrm{H}$ $\operatorname{NMR}\left(400 \mathrm{MHz}, \mathrm{CDCl}_{3}\right) \delta 8.00-7.98(\mathrm{~m}, 1 \mathrm{H}), 7.49-7.46(\mathrm{~m}, 3 \mathrm{H})$, $7.26-7.24(\mathrm{~m}, 2 \mathrm{H}), 7.18-7.15(\mathrm{~m}, 3 \mathrm{H}), 6.84-6.80(\mathrm{~m}, 2 \mathrm{H}), 6.72-$ 
$6.62(\mathrm{~m}, 4 \mathrm{H}), 4.76(\mathrm{~d}, J=8.4 \mathrm{~Hz}, 1 \mathrm{H}), 4.44\left(\mathrm{dd}, J=8.4 \mathrm{~Hz}, J^{\prime}=6.9\right.$ $\mathrm{Hz}, 1 \mathrm{H}), 4.02(\mathrm{q}, J=6.8 \mathrm{~Hz}, 1 \mathrm{H}), 3.71(\mathrm{~s}, 3 \mathrm{H}), 2.98-2.91(\mathrm{~m}, 2 \mathrm{H})$; ${ }^{13} \mathrm{C}\left\{{ }^{1} \mathrm{H}\right\}$ NMR $\left(101 \mathrm{MHz}, \mathrm{CDCl}_{3}\right) \delta 212.6,194.8,139.8,136.7$, $133.8,130.7,129.6$ (2C), 129.2 (2C), 128.8 (2C), 128.4, 128.3 (2C), 128.2 (2C), 128.0, 126.9, 113.7 (2C), 61.6, 50.5, 45.5, 44.9; ATRFTIR $\nu 2934,1741,1608,1570,1509,1246 \mathrm{~cm}^{-1}$; HRMS (ESI) $\mathrm{m} / z$ calcd for $\mathrm{C}_{25} \mathrm{H}_{22} \mathrm{O}_{3} \mathrm{Na}[\mathrm{M}+\mathrm{Na}]^{+}$393.1461, found 393.1467.

Bromination of $\mathbf{2 a}$ with Pyridinium Tribromide. Diketo compound 2a (210 mg, $0.62 \mathrm{mmol}, 1$ equiv) was dissolved in DCM $(18 \mathrm{~mL})$ and cooled to $0{ }^{\circ} \mathrm{C}$. Then $\mathrm{PyrHBr} \cdot \mathrm{Br}_{2}(220 \mathrm{mg}, 90 \%$, $0.62 \mathrm{mmol}, 1$ equiv) was added at once while the reaction mixture was vigorously stirred. The reaction mixture was then stirred overnight at room temperature. A saturated solution of $\mathrm{NaHCO}_{3}$ was added; the organic phase was extracted and washed with water and dried, and the solvent was removed in vacuo. The crude mixture was purified by automated column chromatography with a $98: 2$ petroleum ether/ EtOAc eluent, yielding product $\mathbf{4}$ as a pale orange solid.

(5Z)-2-Bromo-5-[hydroxy(phenyl)methylidene]-3,4-diphenylcyclopentan-1-one (4). Yield $250 \mathrm{mg}(97 \%) ; \mathrm{mp} 120-122{ }^{\circ} \mathrm{C}$ (from $\mathrm{MeOH})$; >1:20 keto:enol; ${ }^{1} \mathrm{H}$ NMR (400 MHz, $\left.\mathrm{CDCl}_{3}\right) \delta 7.58-7.52$ (m, 2H), 7.45-7.38 (m, 1H), 7.30-7.26 (m, 2H), 7.20-7.09 (m, $6 \mathrm{H}), 6.77-6.74(\mathrm{~m}, 4 \mathrm{H}), 5.22\left(\mathrm{dd}, J=12.3 \mathrm{~Hz}, J^{\prime}=1.2 \mathrm{~Hz}, 1 \mathrm{H}\right), 4.43$ $(\mathrm{d}, J=7.6 \mathrm{~Hz}, 1 \mathrm{H}), 3.99\left(\mathrm{dd}, J=12.3 \mathrm{~Hz}, J^{\prime}=7.6 \mathrm{~Hz}, 1 \mathrm{H}\right) ;{ }^{13} \mathrm{C}\left\{{ }^{1} \mathrm{H}\right\}$ NMR $\left(101 \mathrm{MHz}, \mathrm{CDCl}_{3}\right) \delta 200.8,173.2,139.5,136.0,133.1,131.9$, 128.6 (2C), 128.5 (4C), 128.3 (2C), 128.2 (4C), 127.4, 127.2, 110.3, 58.4, 52.3, 51.2; ATR-FTIR $\nu$ 3058, 1637, 1591, 1567, 1491, 1447, 1356, 1142, $1076 \mathrm{~cm}^{-1}$; HRMS (ESI) $m / z$ calcd for $\mathrm{C}_{24} \mathrm{H}_{20} \mathrm{BrO}_{2}[\mathrm{M}$ $+\mathrm{H}]^{+} 419.0641$, found 419.0635 .

Bromination of $2 a$ with $\mathrm{N}$-Bromosuccinimide. According to the published procedure, ${ }^{52}$ a solution of NBS $(26 \mathrm{mg}, 0.15 \mathrm{mmol}, 1$ equiv) in DCM (2 mL) was added to a solution of 1,3-diketone $2 \mathrm{a}$ ( $50 \mathrm{mg}, 0.15 \mathrm{mmol}, 1$ equiv) at rt. The reaction mixture was allowed to stir at room temperature for $6 \mathrm{~h}$ until full conversion was achieved. Then the reaction mixture was directly purified by automated column chromatography with a petroleum ether/EtOAc gradient (98:2 to 97:3), yielding the desired compound $\mathbf{5}$ as a single diastereoisomer as an off-white oil that was crystallized from methanol to give a pale offwhite solid.

2-Benzoyl-2-bromo-3,4-diphenylcyclopentan-1-one (5). Yield 52 $\mathrm{mg}(84 \%) ; \mathrm{mp} 112-115^{\circ} \mathrm{C}$ (from $\left.\mathrm{MeOH}\right) ;{ }^{1} \mathrm{H}$ NMR $(400 \mathrm{MHz}$, $\left.\mathrm{CDCl}_{3}\right) \delta 7.93-7.82(\mathrm{~m}, 2 \mathrm{H}), 7.45-7.36(\mathrm{~m}, 1 \mathrm{H}), 7.30-7.26(\mathrm{~s}$, $2 \mathrm{H}), 7.11-7.08(\mathrm{~m}, 3 \mathrm{H}), 6.98-6.81(\mathrm{~m}, 5 \mathrm{H}), 6.64-6.56(\mathrm{~m}, 2 \mathrm{H})$, $4.64-4.52(\mathrm{~m}, 1 \mathrm{H}), 4.41\left(\mathrm{dd}, J=6.5 \mathrm{~Hz}, J^{\prime}=1.3 \mathrm{~Hz}, 1 \mathrm{H}\right), 3.11(\mathrm{dd}, J$ $\left.=19.1 \mathrm{~Hz}, J^{\prime}=13.3 \mathrm{~Hz}, 1 \mathrm{H}\right), 2.92\left(\mathrm{ddd}, J=19.1 \mathrm{~Hz}, J^{\prime}=7.9 \mathrm{~Hz}, J^{\prime \prime}=\right.$ $1.4 \mathrm{~Hz}, 1 \mathrm{H}) ;{ }^{13} \mathrm{C}\left\{{ }^{1} \mathrm{H}\right\}$ NMR (101 MHz, $\left.\mathrm{CDCl}_{3}\right) \delta$ 204.4, 190.9, $137.2,135.5,135.1,132.9,129.4,129.1,128.2,128.0,127.9,127.4$, 127.1, 77.2, 63.3, 61.6, 42.6, 39.1; ATR-FTIR $\nu$ 3029, 1757, 1664, $1596,1447,1252,1234,1142,1072 \mathrm{~cm}^{-1}$; HRMS (ESI) $\mathrm{m} / z$ calcd for $\mathrm{C}_{24} \mathrm{H}_{20} \mathrm{BrO}_{2}[\mathrm{M}+\mathrm{H}]^{+} 419.0641$, found 419.0631 .

Synthesis of 6 . To diketo compound $2 \mathrm{a}(60 \mathrm{mg}, 0.176 \mathrm{mmol}, 1$ equiv) in DCM (3 mL) was added at $\mathrm{rt}$ a solution of $\mathrm{N}$ (phenylseleno)phthalimide ( $90 \mathrm{mg}, 90 \%, 0.26 \mathrm{mmol}, 1.5$ equiv). The reaction mixture stirred for $3 \mathrm{~h}$ until full conversion was achieved, and the solvent was removed in vacuo. The crude mixture was then dissolved in EtOAc $(6 \mathrm{~mL})$, and hydrogen peroxide $(2 \mathrm{~mL}$ of $30 \%$ in water) was added. After $3 \mathrm{~h}$, full conversion was achieved, the reaction mixture was diluted with water $(1 \mathrm{~mL})$, and the organic phase was collected and washed with saturated $\mathrm{NaHCO}_{3}$ and water and dried over $\mathrm{MgSO}_{4}$. After filtration and evaporation of the solvent, the crude product was separated by automated column chromatography with a petroleum ether/EtOAc gradient (93:7 to 85:15), yielding product 6 as a transparent oil.

2-Benzoyl-3,4-diphenylcyclopent-2-en-1-one (6). Yield $42 \mathrm{mg}$ (70\%); ${ }^{1} \mathrm{H}$ NMR $\left(400 \mathrm{MHz}, \mathrm{CDCl}_{3}\right) \delta 7.94-7.84(\mathrm{~m}, 1 \mathrm{H}), 7.59-$ $7.49(\mathrm{~m}, 1 \mathrm{H}), 7.44-7.40(\mathrm{~m}, 2 \mathrm{H}), 7.31-7.13(\mathrm{~m}, 10 \mathrm{H}), 4.80(\mathrm{dd}, J=$ $\left.7.4 \mathrm{~Hz}, J^{\prime}=2.3 \mathrm{~Hz}, 1 \mathrm{H}\right), 3.30\left(\mathrm{dd}, J=19.0 \mathrm{~Hz}, J^{\prime}=7.4 \mathrm{~Hz}, 1 \mathrm{H}\right), 2.67$ $\left(\mathrm{dd}, J=19.0 \mathrm{~Hz}, J^{\prime}=2.3 \mathrm{~Hz}, 1 \mathrm{H}\right) ;{ }^{13} \mathrm{C}\left\{{ }^{1} \mathrm{H}\right\} \operatorname{NMR}(101 \mathrm{MHz}$, $\left.\mathrm{CDCl}_{3}\right) \delta 204.2,194.6,172.8,141.5,136.1,134.4,134.2,130.8,129.5$ (2C), 129.3 (2C), 128.9 (2C), 128.7 (4C), 127.5 (2C), 127.3, 123.7, 47.3, 46.3; ATR-FTIR $\nu 3057,1690,1654,1597,1579,1497,1331$,
1236, 1174, $1052 \mathrm{~cm}^{-1}$; HRMS (ESI) $m / z$ calcd for $\mathrm{C}_{24} \mathrm{H}_{19} \mathrm{O}_{2}[\mathrm{M}+$ $\mathrm{H}]^{+}$339.1385, found 339.1389 .

Hydroxylation of $2 a$. To the mixture of $2 \mathrm{a}(120 \mathrm{mg}, 0.35 \mathrm{mmol}, 1$ equiv) and $0.5 \mathrm{M}$ aqueous $\mathrm{NaHCO}_{3}(0.7 \mathrm{~mL}, 1$ equiv) in DCM (4 $\mathrm{mL}$ ) was added $m$-CPBA (198 mg, $77 \%, 0.88 \mathrm{mmol}, 2.5$ equiv) portionwise at ambient temperature. After $3 \mathrm{~h}$, full conversion was achieved according to TLC analysis. The excess of peracid was quenched by adding $15 \%$ aqueous $\mathrm{Na}_{2} \mathrm{SO}_{3}(3 \mathrm{~mL})$, and the resulting mixture was stirred for an additional $1 \mathrm{~h}$ at room temperature. The organic layer was then separated, washed with brine $(5 \mathrm{~mL})$, dried over $\mathrm{MgSO}_{4}$, and filtered and concentrated in vacuo. The residue was purified by automated column chromatography with a petroleum ether/EtOAc gradient (98:2 to $85: 15$ ), yielding product 7 as a single diastereoisomer as a thick transparent oil.

2-Benzoyl-2-hydroxy-3,4-diphenylcyclopentan-1-one (7). Yield $56 \mathrm{mg}(45 \%) ;{ }^{1} \mathrm{H}$ NMR (400 MHz, $\left.\mathrm{CDCl}_{3}\right) \delta 7.94-7.63(\mathrm{~m}, 2 \mathrm{H})$, 7.42-7.34 (m, 1H), 7.27-7.19 (m, 2H), 7.15-7.04 (m, 3H), 7.00$6.97(\mathrm{~m}, 2 \mathrm{H}), 6.87-6.84(\mathrm{~m}, 3 \mathrm{H}), 6.76-6.73(\mathrm{~m}, 2 \mathrm{H}), 4.32(\mathrm{dt}, J=$ $\left.14.3 \mathrm{~Hz}, J^{\prime}=7.6 \mathrm{~Hz}, 1 \mathrm{H}\right), 4.19\left(\mathrm{dd}, J=8.0 \mathrm{~Hz}, J^{\prime}=1.2 \mathrm{~Hz}, 1 \mathrm{H}\right), 3.50$ $\left(\mathrm{dd}, J=17.8 \mathrm{~Hz}, J^{\prime}=13.7 \mathrm{~Hz}, 1 \mathrm{H}\right), 2.95\left(\mathrm{ddd}, J=17.8 \mathrm{~Hz}, J^{\prime}=7.3\right.$ $\left.\mathrm{Hz}, J^{\prime \prime}=1.4 \mathrm{~Hz}, 1 \mathrm{H}\right) ;{ }^{13} \mathrm{C}\left\{{ }^{1} \mathrm{H}\right\} \operatorname{NMR}\left(101 \mathrm{MHz}, \mathrm{CDCl}_{3}\right) \delta 213.5$, 198.4, 138.1, 135.1, 135.1, 133.1, 130.4 (2C), 129.9 (2C), 128.2 (2C), 128.0 (4C), 127.9 (2C), 127.0, 126.7, 87.8, 60.3, 42.8, 41.6; ATR-FTIR $\nu 3396,3029,1748,1665,1596,1448,1251,1039 \mathrm{~cm}^{-1}$; HRMS (ESI) $m / z$ calcd for $\mathrm{C}_{24} \mathrm{H}_{21} \mathrm{O}_{3}[\mathrm{M}+\mathrm{H}]^{+} 357.1485$, found 357.1499.

Reaction of $2 a$ with Hydrazine Hydrate. According to the published procedure, ${ }^{56}$ to a sealed vial with $2 \mathrm{a}(168 \mathrm{mg}, 0.49 \mathrm{mmol}, 1$ equiv) in $\mathrm{EtOH}(4 \mathrm{~mL})$ was added hydrazine hydrate $(37 \mu \mathrm{L}, 65 \%$ in water, 1 equiv). The reaction mixture was then heated on an oil bath to reflux until full conversion was achieved as indicated by TLC. The solvent was then removed in vacuo, and the reaction mixture was then purified by automated column chromatography with a petroleum ether/EtOAc gradient (80:20 to 67:33), yielding pyrazole derivative 8 as a yellow thick oil (110 mg, 66\%).

3,4,5-Triphenyl-2H,4H,5H,6H-cyclopenta[c]pyrazole (8). Yield $110 \mathrm{mg}(66 \%) ;{ }^{1} \mathrm{H}$ NMR (400 $\left.\mathrm{MHz}, \mathrm{CDCl}_{3}\right) \delta 10.19$ (bs, $\left.1 \mathrm{H}\right)$, 7.54-7.37 (m, 2H), 7.28-7.19 (m, 3H), 7.13-7.06 (m, 3H), 7.03$6.99(\mathrm{~m}, 3 \mathrm{H}), 6.88-6.86(\mathrm{~m}, 2 \mathrm{H}), 6.74-6.65(\mathrm{~m}, 2 \mathrm{H}), 4.57(\mathrm{~d}, J=$ $7.8 \mathrm{~Hz}, 1 \mathrm{H}), 4.47\left(\mathrm{dtd}, J=10.2 \mathrm{~Hz}, J^{\prime}=7.7 \mathrm{~Hz}, J^{\prime \prime}=2.1 \mathrm{~Hz}, 1 \mathrm{H}\right)$, $3.24\left(\mathrm{dd}, J=15.3 \mathrm{~Hz}, J^{\prime}=10.9 \mathrm{~Hz}, 1 \mathrm{H}\right), 3.07\left(\mathrm{ddd}, J=15.4 \mathrm{~Hz}, J^{\prime}=\right.$ $\left.7.7, J^{\prime \prime}=2.0 \mathrm{~Hz}, 1 \mathrm{H}\right) ;{ }^{13} \mathrm{C}\left\{{ }^{1} \mathrm{H}\right\} \operatorname{NMR}\left(101 \mathrm{MHz}, \mathrm{CDCl}_{3}\right) \delta 140.2$, $140.2,130.3,129.0$ (4C), 128.7 (2C), 128.6 (2C), 128.0 (2C), 127.9 (2C), 127.8 (2C), 126.5, 126.4, 125.8, 125.8, 58.1, 49.4, 29.1; ATRFTIR $\nu 3026,2898,1602,1493,1452,1314,1233,1180,1109 \mathrm{~cm}^{-1}$; HRMS (ESI) $m / z$ calcd for $\mathrm{C}_{24} \mathrm{H}_{21} \mathrm{~N}_{2}[\mathrm{M}+\mathrm{H}]^{+} 337.1699$, found 337.1704 .

Methylation of $2 a$. To the starting 2 a $(0.85 \mathrm{mmol}, 290 \mathrm{mg}$ lequiv) in DMF $(5 \mathrm{~mL})$ under argon was added $\mathrm{K}_{2} \mathrm{CO}_{3}(0.94 \mathrm{mmol}$, $180 \mathrm{mg}, 1.5$ equiv), and then methyl iodide $(0.94 \mathrm{mmol}, 60 \mu \mathrm{L}, 1.1$ equiv) was slowly added. The reaction mixture was then stirred overnight at $60{ }^{\circ} \mathrm{C}$ (oil bath). The next day, the reaction was quenched with water $(5 \mathrm{~mL})$ and the mixture washed with EtOAc (3 $\times 50 \mathrm{~mL})$. The combined organic layer was washed with brine $(2 \times$ $30 \mathrm{~mL}$ ) and dried over $\mathrm{MgSO}_{4}$. After filtration, the solvent was evaporated in vacuo and the crude material was separated by automated column chromatography with a pentane/EtOAc gradient (85:15 to $4: 1$ ), yielding product 9 as a thick transparent oil.

2-Benzoyl-2-methyl-3,4-diphenylcyclopentan-1-one and 2[Methoxy(phenyl)methylidene]-3,4-diphenylcyclopentan-1-one (9a:9b). Yield $210 \mathrm{mg}$ (70\%); 4:1 keto:enol ether. Keto: ${ }^{1} \mathrm{H}$ NMR $\left(400 \mathrm{MHz}, \mathrm{CDCl}_{3}\right) \delta 7.68-7.56(\mathrm{~m}, 2 \mathrm{H}), 7.35-7.31(\mathrm{~m}, 1 \mathrm{H}), 7.29-$ $7.19(\mathrm{~m}, 2 \mathrm{H}), 7.13-7.05(\mathrm{~m}, 4 \mathrm{H}), 6.94-6.87(\mathrm{~m}, 4 \mathrm{H}), 6.77-6.75$ $(\mathrm{m}, 2 \mathrm{H}), 4.15-4.11(\mathrm{~m}, 1 \mathrm{H}), 4.08(\mathrm{t}, J=6.9 \mathrm{~Hz}, 1 \mathrm{H}), 3.28(\mathrm{dd}, J=$ $\left.18.0 \mathrm{~Hz}, J^{\prime}=13.5 \mathrm{~Hz}, 1 \mathrm{H}\right), 2.73\left(\mathrm{dd}, J=18.5 \mathrm{~Hz}, J^{\prime}=6.2 \mathrm{~Hz}, 1 \mathrm{H}\right)$, $1.85(\mathrm{~s}, 3 \mathrm{H}) ;{ }^{13} \mathrm{C}\left\{{ }^{1} \mathrm{H}\right\}$ NMR $\left(101 \mathrm{MHz}, \mathrm{CDCl}_{3}\right) \delta 214.1,199.2$, $137.9,137.2,136.2,132.0,129.5,128.7$ (2C), 128.0 (6C), 127.9 (2C), 127.6 (2C), 126.7, 65.8, 60.2, 43.2, 40.6, 24.7. Enol: ${ }^{1} \mathrm{H}$ NMR $\left(400 \mathrm{MHz}, \mathrm{CDCl}_{3}\right) \delta 7.77-7.69(\mathrm{~m}, 2 \mathrm{H}), 7.47-7.44(\mathrm{~m}, 1 \mathrm{H}), 7.40-$ $7.37(\mathrm{~m}, 2 \mathrm{H}), 7.03-6.97(\mathrm{~m}, 3 \mathrm{H}), 6.87(\mathrm{~s}, 4 \mathrm{H}), 4.58(\mathrm{~d}, J=8.2 \mathrm{~Hz}$, 
$1 \mathrm{H}), 4.01(\mathrm{q}, J=8.8 \mathrm{~Hz}, 1 \mathrm{H}), 3.77(\mathrm{~s}, 3 \mathrm{H}), 3.22-3.15(\mathrm{~m}, 1 \mathrm{H}), 3.01$ $\left(\mathrm{dd}, J=16.8 \mathrm{~Hz}, J^{\prime}=8.1 \mathrm{~Hz}, 1 \mathrm{H}\right) ;{ }^{13} \mathrm{C}\left\{{ }^{1} \mathrm{H}\right\}$ NMR $(101 \mathrm{MHz}$, $\left.\mathrm{CDCl}_{3}\right) \delta 192.4,167.6,140.3,140.1,139.7,131.3,128.6$ (2C), 128.2 (2C), 127.7 (4C), 126.7 (4C), 126.3, 126.1, 116.2, 58.0, 54.4, 46.7, 35.3; ATR-FTIR $\nu 3026,1745,1669,1596,1454,1246 \mathrm{~cm}^{-1}$; HRMS (ESI) $m / z$ calcd for $\mathrm{C}_{25} \mathrm{H}_{23} \mathrm{O}_{2}[\mathrm{M}+\mathrm{H}]^{+} 355.1698$, found 355.1700 .

Aldol Reaction of $2 a$ with Benzophenone. According to the modified procedure, ${ }^{55}$ diisopropylamine $(91 \mu \mathrm{L}, 2.20$ equiv) was dissolved in dry tetrahydrofuran $(5 \mathrm{~mL})$, and after the mixture had cooled to $0{ }^{\circ} \mathrm{C}$, $n$-butyllithium $(260 \mu \mathrm{L}, 2.20$ equiv, $2.5 \mathrm{M}$ solution in $n$-hexane) was added. The reaction mixture was stirred at $\mathrm{rt}$ for 30 min followed by further cooling to $0{ }^{\circ} \mathrm{C}$. Diketone $2 \mathrm{a}(100 \mathrm{mg}, 0.29$ mmol, 1 equiv) was dissolved in dry tetrahydrofuran $(3 \mathrm{~mL})$ and slowly added to the solution of diisopropylamine and $n$-butyllithium, and the mixture was stirred. After $15 \mathrm{~min}$, benzophenone $(65 \mathrm{mg}$, $0.35 \mathrm{mmol}, 1.20$ equiv) was added and the reaction mixture stirred at $0{ }^{\circ} \mathrm{C}$ for $30 \mathrm{~min}$ and then at rt overnight. After $16 \mathrm{~h}$, TLC revealed full conversion; the reaction was then quenched by the addition of a saturated aqueous solution of ammonium chloride $(1 \mathrm{~mL})$ and water $(10 \mathrm{~mL})$, and EtAOc was then added. The organic phase was separated, and the aqueous phase extracted with EtOAc $(2 \times 20 \mathrm{~mL})$. The combined organic phases were washed with brine and dried over $\mathrm{MgSO}_{4}$, and the solvent was evaporated in vacuo. The crude material was purified by automated column chromatography with a petroleum ether/EtOAc gradient (95:5 to 92:8), yielding product 10 as a transparent oil.

(2Z)-2-[Hydroxy(phenyl)methylidene]-5-(hydroxydiphenylmethyl)-3,4-diphenylcyclopentan-1-one (10). Yield $100 \mathrm{mg}(65 \%) ;>1: 20$ keto/enol; ${ }^{1} \mathrm{H}$ NMR $\left(400 \mathrm{MHz}, \mathrm{CDCl}_{3}\right) \delta 7.50$ (ddd, $J=15.3 \mathrm{~Hz}, J^{\prime}$ $\left.=8.2 \mathrm{~Hz}, J^{\prime \prime}=1.2 \mathrm{~Hz}, 4 \mathrm{H}\right), 7.39-7.31(\mathrm{~m}, 1 \mathrm{H}), 7.28-7.18(\mathrm{~m}, 6 \mathrm{H})$, $7.05-6.93(\mathrm{~m}, 5 \mathrm{H}), 6.86-6.60(\mathrm{~m}, 5 \mathrm{H}), 6.33(\mathrm{bs}, 2 \mathrm{H}), 4.36(\mathrm{~d}, J=$ $10.6 \mathrm{~Hz}, 1 \mathrm{H}), 4.25(\mathrm{~d}, J=8.2 \mathrm{~Hz}, 1 \mathrm{H}), 4.04\left(\mathrm{dd}, J=10.6 \mathrm{~Hz}, J^{\prime}=8.1\right.$ $\mathrm{Hz}, 1 \mathrm{H}), 3.87(\mathrm{~s}, 1 \mathrm{H}) ;{ }^{13} \mathrm{C}\left\{{ }^{1} \mathrm{H}\right\}$ NMR $\left(101 \mathrm{MHz}, \mathrm{CDCl}_{3}\right) \delta 208.7$, $172.9,146.1,143.9,141.4,139.5,133.8,131.4,128.6$ (4C), 128.2 (4C), 128.1 (2C), 127.9 (2C), 127.5 (2C), 127.3 (2C), 127.2, 127.0 (4C), 126.8, 126.5, 125.4, 113.5, 79.7, 57.7, 51.0, 50.8; ATR-FTIR $\nu$ $3061,3028,2956,1738,1603,1568,1492,1447,1365,1269,1218$, $1154 \mathrm{~cm}^{-1}$; HRMS (ESI) $\mathrm{m} / z$ calcd for $\mathrm{C}_{37} \mathrm{H}_{29} \mathrm{O}_{3}[\mathrm{M}-\mathrm{H}]^{-}$ 521.2117, found 521.2119.

Selenylation of $2 \boldsymbol{a}$. According to the modified procedure, ${ }^{55}$ diisopropylamine (91 $\mu \mathrm{L}, 2.20$ equiv) was dissolved in dry tetrahydrofuran $(5 \mathrm{~mL})$. After the mixture had cooled to $0{ }^{\circ} \mathrm{C}$, $n$ butyllithium ( $260 \mu \mathrm{L}, 2.20$ equiv, $2.5 \mathrm{M}$ solution in $n$-hexane) was added and the reaction mixture was stirred at room temperature for $30 \mathrm{~min}$. Then after the mixture had again cooled to $0{ }^{\circ} \mathrm{C}$, diketone 2a (100 mg, $0.29 \mathrm{mmol}, 1$ equiv) was dissolved in dry tetrahydrofuran ( 3 $\mathrm{mL}$ ) and slowly added to the solution of diisopropylamine and $n$ butyllithium. The reaction mixture was stirred for $15 \mathrm{~min}$ followed by an addition of $\mathrm{N}$-(phenylseleno)phthalimide $(120 \mathrm{mg}, 1.20$ equiv); the subsequent mixture was stirred at $0{ }^{\circ} \mathrm{C}$ for $30 \mathrm{~min}$ and then at $\mathrm{rt}$ for an additional $60 \mathrm{~min}$ when, according to TLC, full conversion was achieved. The reaction was then quenched by the addition of a saturated aqueous solution of $\mathrm{NH}_{4} \mathrm{Cl}(1 \mathrm{~mL})$ and water $(10 \mathrm{~mL})$, and EtAOc $(20 \mathrm{~mL})$ was then added. The organic phase was separated, and the aqueous phase extracted with EtOAc $(2 \times 20 \mathrm{~mL})$. The combined organic phases were washed with brine and dried over $\mathrm{MgSO}_{4}$, and the solvent was evaporated in vacuo. The crude material was purified by automated column chromatography with a petroleum ether/EtOAc gradient (98:2 to 92:8), yielding product 11 as single diastereoisomer in the form of a thick yellow oil.

(2Z)-2-[Hydroxy(phenyl)methylidene]-3,4-diphenyl-5(phenylselanyl)cyclopentan-1-one (11). Yield $110 \mathrm{mg}(76 \%)$ as a thick yellow oil; 1:20 keto/enol. Enol: ${ }^{1} \mathrm{H}$ NMR $\left(400 \mathrm{MHz}, \mathrm{CDCl}_{3}\right) \delta$ $7.62-7.53(\mathrm{~m}, 2 \mathrm{H}), 7.52-7.49$ (m, 2H), 7.39-7.26 (m, 2H), 7.28$7.18(\mathrm{~m}, 4 \mathrm{H}), 7.14-7.01(\mathrm{~m}, 6 \mathrm{H}), 6.75-6.68(\mathrm{~m}, 2 \mathrm{H}), 6.67-6.59$ $(\mathrm{m}, 2 \mathrm{H}), 4.42(\mathrm{~d}, J=12.1 \mathrm{~Hz}, 1 \mathrm{H}), 4.33(\mathrm{~d}, J=7.6 \mathrm{~Hz}, 1 \mathrm{H}), 3.66$ $\left(\mathrm{dd}, J=12.1 \mathrm{~Hz}, J^{\prime}=7.6 \mathrm{~Hz}, 1 \mathrm{H}\right) ;{ }^{13} \mathrm{C}\left\{{ }^{1} \mathrm{H}\right\} \operatorname{NMR}(101 \mathrm{MHz}$, $\left.\mathrm{CDCl}_{3}\right) \delta 205.2,172.3,140.2,137.6,136.50(2 \mathrm{C}), 133.7,131.5,129.0$ (2C), 128.6, 128.5 (6C), 128.3 (4C), 128.0 (2C), 126.9 (2C), 126.8, 111.6, 54.5, 51.3, 50.5. Keto: ${ }^{1} \mathrm{H}$ NMR (400 MHz, $\left.\mathrm{CDCl}_{3}\right) \delta 8.10-$ $8.04(\mathrm{~m}, 2 \mathrm{H}), 7.76\left(\mathrm{dd}, J=8.2 \mathrm{~Hz}, J^{\prime}=1.3 \mathrm{~Hz}, 1 \mathrm{H}\right), 7.68-7.61(\mathrm{~m}$, $4 \mathrm{H}), 7.35-7.28(\mathrm{~m}, 2 \mathrm{H}), 7.26-7.20(\mathrm{~m}, 2 \mathrm{H}), 7.18-7.12(\mathrm{~m}, 3 \mathrm{H})$, $7.08-7.04(\mathrm{~m}, 2 \mathrm{H}), 6.80-6.75(\mathrm{~m}, 4 \mathrm{H}), 4.86\left(\mathrm{dd}, J=8.1 \mathrm{~Hz}, J^{\prime}=1.2\right.$ $\mathrm{Hz}, 1 \mathrm{H}), 4.70(\mathrm{t}, J=7.6 \mathrm{~Hz}, 1 \mathrm{H}), 4.29\left(\mathrm{dd}, J=5.6 \mathrm{~Hz}, J^{\prime}=1.3 \mathrm{~Hz}\right.$, $1 \mathrm{H}), 3.97-3.89(\mathrm{~m}, 1 \mathrm{H}) ;{ }^{13} \mathrm{C}\left\{{ }^{1} \mathrm{H}\right\} \mathrm{NMR}\left(101 \mathrm{MHz}, \mathrm{CDCl}_{3}\right) \delta 206.9$, $192.4,136.5$ (2C), 136.1 (2C), 133.7, 131.5, 130.0, 129.3, 129.0 (2C), 128.6 (2C), 128.5 (2C), 128.5 (4C), 128.3 (2C), 128.2 (2C), 127.1 (2C), 126.9, 60.6, 52.2, 48.91; ATR-FTIR $\nu$ 3059, 3028, 1719, $1604,1592,1568,1493,1360,1260,1221,1150,1071 \mathrm{~cm}^{-1}$; HRMS (ESI) $m / z$ calcd for $\mathrm{C}_{30} \mathrm{H}_{25} \mathrm{O}_{2} \mathrm{Se}[\mathrm{M}+\mathrm{H}]^{+}$497.1014, found 497.1019.

Elimination of Brominated Diketo Compound 4. 1,4Diazabicyclo[2.2.2] octane (DABCO) (0.49 mmol, $55 \mathrm{mg}, 2$ equiv) was added to a stirred solution of brominated diketone $4(0.24 \mathrm{mmol}$, $100 \mathrm{mg}, 1$ equiv) in THF $(5 \mathrm{~mL})$ at rt. Full conversion was achieved after $2 \mathrm{~h}$ (TLC monitoring). The reaction mixture was evaporated and purified by automated column chromatography with a pentane/ EtOAc gradient (9:1 to 5:1), yielding product 12 as a thick orange oil.

5-Benzoyl-3,4-diphenylcyclopent-2-en-1-one (12). Yield $70 \mathrm{mg}$ $(85 \%)$; >2:1 keto/enol. Keto: ${ }^{1} \mathrm{H}$ NMR $\left(400 \mathrm{MHz}, \mathrm{CDCl}_{3}\right) \delta 8.13-$ 8.01 (m, 2H), 7.66-7.57 (m, 2H), 7.55-7.44 (m, 3H), 7.41-7.24 (m, 4H), 7.25-7.16 (m, 3H), 7.00-6.89 (m, 1H), $6.69(\mathrm{~d}, J=1.4 \mathrm{~Hz}$, $1 \mathrm{H}), 5.34(\mathrm{t}, J=1.7 \mathrm{~Hz}, 1 \mathrm{H}), 4.61(\mathrm{~d}, J=2.0 \mathrm{~Hz}, 1 \mathrm{H}) ;{ }^{13} \mathrm{C}\left\{{ }^{1} \mathrm{H}\right\}$ NMR $\left(101 \mathrm{MHz}, \mathrm{CDCl}_{3}\right) \delta 200.9,193.2,175.9,141.3,136.2,133.7$, $132.9,131.4,130.6,130.2$ (2C), 129.4 (2C), 128.9 (2C), 128.7 (2C), 128.4 (2C), 127.6 (2C), 126.5, 67.1, 50.4. Enol: ${ }^{1} \mathrm{H}$ NMR $(400 \mathrm{MHz}$, $\left.\mathrm{CDCl}_{3}\right) \delta 8.12-8.08(\mathrm{~m}, 2 \mathrm{H}), 7.60-7.57(\mathrm{~m}, 2 \mathrm{H}), 7.55-7.50(\mathrm{~m}$, $2 \mathrm{H}), 7.41-7.24(\mathrm{~m}, 5 \mathrm{H}), 7.24-7.18(\mathrm{~m}, 2 \mathrm{H}), 7.00-6.91(\mathrm{~m}, 2 \mathrm{H})$, $6.87(\mathrm{~d}, J=1.2 \mathrm{~Hz}, 0 \mathrm{H}), 5.26(\mathrm{~d}, J=1.3 \mathrm{~Hz}, 1 \mathrm{H}) ;{ }^{13} \mathrm{C}\left\{{ }^{1} \mathrm{H}\right\} \mathrm{NMR}$ $\left(101 \mathrm{MHz}, \mathrm{CDCl}_{3}\right) \delta 199.8,169.9,168.7,138.8,134.2,133.2,130.5$, 128.8 (2C), 128.3 (4C), 128.1 (2C), 128.0 (2C), 127.9 (2C), 127.5 (2C), 126.8, 116.8, 50.8; ATR-FTIR $\nu$ 3060, 1690, 1666, 1596, 1570, $1493,1446,1176,1148,1000 \mathrm{~cm}^{-1}$; HRMS (ESI) $\mathrm{m} / z$ calcd for $\mathrm{C}_{24} \mathrm{H}_{19} \mathrm{O}_{2}[\mathrm{M}+\mathrm{H}]^{+}$339.1380, found 339.1380.

General Procedure for Sample Preparation and Crystal Measurement. Single crystals of products $2 \mathrm{a}, \mathbf{4}$, and 5 were prepared by volatilization using a mixture of methanol and dichloromethane as a solvent. Suitable crystals were selected and collected on a Bruker D8 VENTURE Kappa Duo PHOTONIII instrument by an I $\mu \mathrm{S}$ microfocus sealed tube with Mo $\mathrm{K} \alpha(\lambda=0.71073)(5)$ or $\mathrm{Cu} \mathrm{K} \alpha$ $(\lambda=1.54178 \AA)(2 \mathrm{a}$ and 4$)$ radiation at a low temperature of $120 \mathrm{~K}$.

\section{ASSOCIATED CONTENT}

\section{Supporting Information}

The Supporting Information is available free of charge at https://pubs.acs.org/doi/10.1021/acs.joc.1c00445.

NMR spectra for all new compounds (PDF)

\section{Accession Codes}

CCDC 2063174-2063176 contain the supplementary crystallographic data for this paper. These data can be obtained free of charge via www.ccdc.cam.ac.uk/data_request/cif, or by emailing data_request@ccdc.cam.ac.uk, or by contacting The Cambridge Crystallographic Data Centre, 12 Union Road, Cambridge CB2 1EZ, UK; fax: +44 1223336033.

\section{AUTHOR INFORMATION}

\section{Corresponding Author}

Henrik Sundén - Chalmers University of Technology, Department of Chemistry and Chemical Engineering, 41296 Gothenburg, Sweden; University of Gothenburg, Department of Chemistry and Molecular Biology, SE-412 96 Gothenburg, Sweden; @ orcid.org/0000-0001-6202-7557;

Email: henrik.sunden@chem.gu.se 


\section{Authors}

Martin Kamlar - Chalmers University of Technology, Department of Chemistry and Chemical Engineering, 41296 Gothenburg, Sweden; Department of Organic Chemistry, Faculty of Science, Charles University, 12843 Prague, Czech Republic

Elin Henriksson - Chalmers University of Technology, Department of Chemistry and Chemical Engineering, 41296 Gothenburg, Sweden

Ivana Císarová - Department of Inorganic Chemistry, Faculty of Science, Charles University, 12843 Prague, Czech Republic

Marcus Malo - University of Gothenburg, Department of Chemistry and Molecular Biology, SE-412 96 Gothenburg, Sweden

Complete contact information is available at: https://pubs.acs.org/10.1021/acs.joc.1c00445

\section{Notes}

The authors declare no competing financial interest.

\section{ACKNOWLEDGMENTS}

This work was supported by grants from the Swedish Research Council Formas (2019-00699). M.K. thanks the Operational Programme Research, Development and Education (Project Improvement of internationalization in the field of research and development) at Charles University through the support of quality projects MSCA-IF (CZ.02.2.69/0.0/0.0/17_050/ 0008466) for postdoctoral funding.

\section{REFERENCES}

(1) Jeffrey, J. L.; Sarpong, R. Concise Synthesis of Pauciflorol F Using a Larock Annulation. Org. Lett. 2009, 11 (23), 5450-5453.

(2) Yang, Y.; Philips, D.; Pan, S. A Concise Synthesis of Paucifloral F and Related Indanone Analogues via Palladium-Catalyzed $\alpha$ Arylation. J. Org. Chem. 2011, 76 (6), 1902-1905.

(3) Lee, B. H.; Choi, Y. L.; Shin, S.; Heo, J.-N. Stereoselective Palladium-Catalyzed $\alpha$-Arylation of 3-Aryl-1-Indanones: An Asymmetric Synthesis of (+)-Pauciflorol F. J. Org. Chem. 2011, 76 (16), 6611-6618.

(4) Kerr, D. J.; Miletic, M.; Manchala, N.; White, J. M.; Flynn, B. L. Asymmetric Synthesis of (+)- and (-)-Pauciflorol F: Confirmation of Absolute Stereochemistry. Org. Lett. 2013, 15 (16), 4118-4121.

(5) Li, C.; Xu, X.; Tao, Z.; Wang, X. J.; Pan, Y. Resveratrol Dimers, Nutritional Components in Grape Wine, Are Selective ROS Scavengers and Weak Nrf2 Activators. Food Chem. 2015, 173, 218223.

(6) Tang, M.-L.; Peng, P.; Liu, Z.-Y.; Zhang, J.; Yu, J.-M.; Sun, X. Sulfoxide-Based Enantioselective Nazarov Cyclization: Divergent Syntheses of (+)-Isopaucifloral F, (+)-Quadrangularin A, and (+)-Pallidol. Chem. - Eur. J. 2016, 22 (41), 14535-14539.

(7) Faiz, S.; Yousaf, M.; Zahoor, A. F.; Naqvi, S. A. R.; Irfan, A.; Zaman, G. Synthetic Strategies toward the Synthesis of Polyphenolic Natural Products: Pauciflorol F and Isopaucifloral F: A Review. Synth. Commun. 2017, 47 (12), 1121-1135.

(8) Coggon, P.; King, T. J.; Wallwork, S. C. The Structure of Hopeaphenol. Chem. Commun. 1966, 13, 439-440.

(9) Tanaka, T.; Ito, T.; Iinuma, M.; Ohyama, M.; Ichise, M.; Tateishi, Y. Stilbene Oligomers in Roots of Sophora Davidii. Phytochemistry 2000, 53 (8), 1009-1014.

(10) Klotter, F.; Studer, A. Total Synthesis of Resveratrol-Based Natural Products Using a Palladium-Catalyzed Decarboxylative Arylation and an Oxidative Heck Reaction. Angew. Chem., Int. Ed. 2014, 53 (9), 2473-2476.
(11) He, S.; Wu, B.; Pan, Y.; Jiang, L. Stilbene Oligomers from Parthenocissus Laetevirens: Isolation, Biomimetic Synthesis, Absolute Configuration, and Implication of Antioxidative Defense System in the Plant. J. Org. Chem. 2008, 73 (14), 5233-5241.

(12) Ito, T.; Tanaka, T.; Inuma, M.; Nakaya, K.; Takahashi, Y.; Sawa, R.; Murata, J.; Darnaedi, D. Three New Resveratrol Oligomers from the Stem Bark of Vatica Pauciflora. J. Nat. Prod. 2004, 67 (6), 932-937.

(13) Vitrac, X.; Bornet, A.; Vanderlinde, R.; Valls, J.; Richard, T.; Delaunay, J.-C.; Mérillon, J.-M.; Teissédre, P.-L. Determination of Stilbenes $(\delta$-Viniferin, Trans-Astringin, Trans-Piceid, Cis- and TransResveratrol, $\varepsilon$-Viniferin) in Brazilian Wines. J. Agric. Food Chem. 2005 53 (14), 5664-5669.

(14) Sharp, H.; Hollinshead, J.; Bartholomew, B. B.; Oben, J.; Watson, A.; Nash, R. J. Inhibitory Effects of Cissus Quadrangularis L. Derived Components on Lipase, Amylase and $\alpha$-Glucosidase Activity in Vitro. Nat. Prod. Commun. 2007, 2 (8), 1934578X0700200.

(15) Wada, S.; Yasui, Y.; Hitomi, T.; Tanaka, R. Structures and Radical-Scavenging Activities of Phenolic Constituents from the Bark of Picea Jezoensis Var. Jezoensis. J. Nat. Prod. 2007, 70 (10), 16051610.

(16) Snyder, S. A.; Zografos, A. L.; Lin, Y. Total Synthesis of Resveratrol-Based Natural Products: A Chemoselective Solution. Angew. Chem., Int. Ed. 2007, 46 (43), 8186-8191.

(17) Snyder, S. A.; Breazzano, S. P.; Ross, A. G.; Lin, Y.; Zografos, A. L. Total Synthesis of Diverse Carbogenic Complexity within the Resveratrol Class from a Common Building Block. J. Am. Chem. Soc. 2009, 131 (5), 1753-1765.

(18) Zhong, C.; Liu, X.-H.; Chang, J.; Yu, J.-M.; Sun, X. Inhibitory Effect of Resveratrol Dimerized Derivatives on Nitric Oxide Production in Lipopolysaccharide-Induced RAW 264.7 Cells. Bioorg. Med. Chem. Lett. 2013, 23 (15), 4413-4418.

(19) Papastamoulis, Y.; Richard, T.; Nassra, M.; Badoc, A.; Krisa, S.; Harakat, D.; Monti, J.-P.; Mérillon, J.-M.; Waffo-Teguo, P. Viniphenol A, a Complex Resveratrol Hexamer from Vitis Vinifera Stalks: Structural Elucidation and Protective Effects against Amyloid- $\beta$ Induced Toxicity in PC12 Cells. J. Nat. Prod. 2014, 77 (2), 213-217.

(20) Gheewala, C. D.; Radtke, M. A.; Hui, J.; Hon, A. B.; Lambert, T. H. Methods for the Synthesis of Functionalized Pentacarboxycyclopentadienes. Org. Lett. 2017, 19 (16), 4227-4230.

(21) Rodrigo, C. M.; Cencic, R.; Roche, S. P.; Pelletier, J.; Porco, J. A. Synthesis of Rocaglamide Hydroxamates and Related Compounds as Eukaryotic Translation Inhibitors: Synthetic and Biological Studies. J. Med. Chem. 2012, 55 (1), 558-562.

(22) Wu, T. S.; Liou, M. J.; Kuoh, C. S.; Teng, C. M.; Nagao, T.; Lee, K. H. Cytotoxic and Antiplatelet Aggregation Principles from Aglaia Elliptifolia. J. Nat. Prod. 1997, 60 (6), 606-608.

(23) Adams, T. E.; El Sous, M.; Hawkins, B. C.; Hirner, S.; Holloway, G.; Khoo, M. L.; Owen, D. J.; Savage, G. P.; Scammells, P. J.; Rizzacasa, M. A. Total Synthesis of the Potent Anticancer Aglaia Metabolites (-)-Silvestrol and (-)-Episilvestrol and the Active Analogue (-)-4-Desmethoxyepisilvestrol. J. Am. Chem. Soc. 2009, 131 (4), 1607-1616.

(24) Zhao, Q.; Abou-Hamdan, H.; Désaubry, L. Recent Advances in the Synthesis of Flavaglines, a Family of Potent Bioactive Natural Compounds Originating from Traditional Chinese Medicine. Eur. J. Org. Chem. 2016, 2016 (36), 5908-5916.

(25) Gerard, B.; Cencic, R.; Pelletier, J.; Porco, J. A. Enantioselective Synthesis of the Complex Rocaglate (-)-Silvestrol. Angew. Chem. 2007, 119 (41), 7977-7980.

(26) Watanabe, T.; Kohzuma, S.; Takeuchi, T.; Otsuka, M.; Umezawa, K. Total Synthesis of ( \pm )-Aglaiastatin, a Novel Bioactive Alkaloid. Chem. Commun. 1998, 10, 1097-1098.

(27) Hwang, B. Y.; Su, B. N.; Chai, H.; Mi, Q.; Kardono, L. B. S.; Afriastini, J. J.; Riswan, S.; Santarsiero, B. D.; Mesecar, A. D.; Wild, R.; Fairchild, C. R.; Vite, G. D.; Rose, W. C.; Farnsworth, N. R.; Cordell, G. A.; Pezzuto, J. M.; Swanson, S. M.; Kinghorn, A. D. Silvestrol and Episilvestrol, Potential Anticancer Rocaglate Derivatives from Aglaia Silvestris. J. Org. Chem. 2004, 69 (10), 3350-3358. 
(28) Zhu, J. Y.; Lavrik, I. N.; Mahlknecht, U.; Giaisi, M.; Proksch, P.; Krammer, P. H.; Li-Weber, M. The Traditional Chinese Herbal Compound Rocaglamide Preferentially Induces Apoptosis in Leukemia Cells by Modulation of Mitogen-Activated Protein Kinase Activities. Int. J. Cancer 2007, 121 (8), 1839-1846.

(29) Astelbauer, F.; Gruber, M.; Brem, B.; Greger, H.; Obwaller, A.; Wernsdorfer, G.; Congpuong, K.; Wernsdorfer, W. H.; Walochnik, J. Activity of Selected Phytochemicals against Plasmodium Falciparum. Acta Trop. 2012, 123 (2), 96-100.

(30) Thuaud, F.; Ribeiro, N.; Gaiddon, C.; Cresteil, T.; Désaubry, L. Novel Flavaglines Displaying Improved Cytotoxicity. J. Med. Chem. 2011, 54 (1), 411-415.

(31) Zhang, W.; Liu, S.; Maiga, R. I.; Pelletier, J.; Brown, L. E.; Wang, T. T.; Porco, J. A. Chemical Synthesis Enables Structural Reengineering of Aglaroxin C Leading to Inhibition Bias for Hepatitis C Viral Infection. J. Am. Chem. Soc. 2019, 141 (3), 1312-1323.

(32) Ribeiro, N.; Thuaud, F.; Bernard, Y.; Gaiddon, C.; Cresteil, T.; Hild, A.; Hirsch, E. C.; Michel, P. P.; Nebigil, C. G.; Désaubry, L. Flavaglines as Potent Anticancer and Cytoprotective Agents. J. Med. Chem. 2012, 55 (22), 10064-10073.

(33) Stone, S. D.; Lajkiewicz, N. J.; Whitesell, L.; Hilmy, A.; Porco, J. A. Biomimetic Kinetic Resolution: Highly Enantio- and Diastereoselective Transfer Hydrogenation of Aglain Ketones to Access Flavagline Natural Products. J. Am. Chem. Soc. 2015, 137 (1), 525530.

(34) Liu, T.; Nair, S. J.; Lescarbeau, A.; Belani, J.; Peluso, S.; Conley, J.; Tillotson, B.; O'Hearn, P.; Smith, S.; Slocum, K.; West, K.; Helble, J.; Douglas, M.; Bahadoor, A.; Ali, J.; McGovern, K.; Fritz, C.; Palombella, V. J.; Wylie, A.; Castro, A. C.; Tremblay, M. R. Synthetic Silvestrol Analogues as Potent and Selective Protein Synthesis Inhibitors. J. Med. Chem. 2012, 55 (20), 8859-8878.

(35) Chan, K.; Robert, F.; Oertlin, C.; Kapeller-Libermann, D.; Avizonis, D.; Gutierrez, J.; Handly-Santana, A.; Doubrovin, M.; Park, J.; Schoepfer, C.; Da Silva, B.; Yao, M.; Gorton, F.; Shi, J.; Thomas, C. J.; Brown, L. E.; Porco, J. A.; Pollak, M.; Larsson, O.; Pelletier, J.; Chio, I. I. C. EIF4A Supports an Oncogenic Translation Program in Pancreatic Ductal Adenocarcinoma. Nat. Commun. 2019, 10 (1), 5151.

(36) Müller, C.; Schulte, F. W.; Lange-Grünweller, K.; Obermann, W.; Madhugiri, R.; Pleschka, S.; Ziebuhr, J.; Hartmann, R. K.; Grünweller, A. Broad-Spectrum Antiviral Activity of the EIF4A Inhibitor Silvestrol against Corona- and Picornaviruses. Antiviral Res. 2018, 150, 123-129.

(37) Biedenkopf, N.; Lange-Grünweller, K.; Schulte, F. W.; Weißer, A.; Müller, C.; Becker, D.; Becker, S.; Hartmann, R. K.; Grünweller, A. The Natural Compound Silvestrol Is a Potent Inhibitor of Ebola Virus Replication. Antiviral Res. 2017, 137, 76-81.

(38) Todt, D.; Moeller, N.; Praditya, D.; Kinast, V.; Friesland, M.; Engelmann, M.; Verhoye, L.; Sayed, I. M.; Behrendt, P.; Dao Thi, V. L.; Meuleman, P.; Steinmann, E. The Natural Compound Silvestrol Inhibits Hepatitis E Virus (HEV) Replication in Vitro and in Vivo. Antiviral Res. 2018, 157, 151-158.

(39) Trost, B. M.; Greenspan, P. D.; Yang, B. V.; Saulnier, M. G. An Unusual Oxidative Cyclization. A Synthesis and Absolute Stereochemical Assignment of (-)-Rocaglamide. J. Am. Chem. Soc. 1990, 112 (24), 9022-9024.

(40) Corey, E. J.; Lee, D. H. Highly Enantioselective and Diastereoselective Ireland-Claisen Rearrangement of Achiral Allylic Esters. J. Am. Chem. Soc. 1991, 113 (10), 4026-4028.

(41) Dobler, M. R.; Bruce, I.; Cederbaum, F.; Cooke, N. G.; Diorazio, L. J.; Hall, R. G.; Irving, E. Total Synthesis of ( \pm -Rocaglamide and Some Aryl Analogues. Tetrahedron Lett. 2001, 42 (47), 8281-8284.

(42) Thede, K.; Diedrichs, N.; Ragot, J. P. Stereoselective Synthesis of ( \pm )-Rocaglaol Analogues. Org. Lett. 2004, 6 (24), 4595-4597.

(43) Diedrichs, N.; Ragot, J. P.; Thede, K. A Highly Efficient Synthesis of Rocaglaols by a Novel $\alpha$-Arylation of Ketones. Eur. J. Org. Chem. 2005, 2005 (9), 1731-1735.
(44) Gerard, B.; Sangji, S.; O’Leary, D. J.; Porco, J. A. Enantioselective Photocycloaddition Mediated by Chiral Brønsted Acids: Asymmetric Synthesis of the Rocaglamides. J. Am. Chem. Soc. 2006, 128 (24), 7754-7755.

(45) Tamiya, M.; Jager, C.; Ohmori, K.; Suzuki, K. Stereoselective Access to Functionalized Dihydrophenanthrenes via Reductive Cyclization of Biaryl Ene-Aldehydes. Synlett 2007, 5, 780-784.

(46) Giese, M. W.; Moser, W. H. Stereoselective Synthesis of the Rocaglamide Skeleton via a Silyl Vinylketene Formation/ $[4+1]$ Annulation Sequence. Org. Lett. 2008, 10 (19), 4215-4218.

(47) Malona, J. A.; Cariou, K.; Frontier, A. J. Nazarov Cyclization Initiated by Peracid Oxidation: The Total Synthesis of $( \pm)$-Rocaglamide. J. Am. Chem. Soc. 2009, 131 (22), 7560-7561.

(48) Ramadhar, T. R.; Kawakami, J.; Lough, A. J.; Batey, R. A. Stereocontrolled Synthesis of Contiguous C(Sp3)-C(Aryl) Bonds by Lanthanide(III)-Catalyzed Domino Aryl-Claisen [3,3]-Sigmatropic Rearrangements. Org. Lett. 2010, 12 (20), 4446-4449.

(49) Wang, X.; Xie, Z.; Fu, B.; Li, N.; Wang, M.; Ma, Y.; Du, F.; Xu, Y.; Qin, Z. Studies on Synthesis and Biological Activity of Oxidative Aglafolin Derivatives. J. Heterocycl. Chem. 2014, 51 (5), 1430-1434.

(50) Zhou, Z.; Tius, M. A. Synthesis of Each Enantiomer of Rocaglamide by Means of a Palladium(0)-Catalyzed Nazarov-Type Cyclization. Angew. Chem., Int. Ed. 2015, 54 (20), 6037-6040.

(51) Zhou, Z.; Dixon, D. D.; Jolit, A.; Tius, M. A. The Evolution of the Total Synthesis of Rocaglamide. Chem. - Eur. J. 2016, 22 (44), 15929-15936.

(52) Ta, L.; Axelsson, A.; Bijl, J.; Haukka, M.; Sundén, H. Ionic Liquids as Precatalysts in the Highly Stereoselective Conjugate Addition of $\alpha, \beta$-Unsaturated Aldehydes to Chalcones. Chem. - Eur. J. 2014, 20 (43), 13889-13893.

(53) Axelsson, A.; Ta, L.; Sundén, H. Ionic Liquids as Carbene Catalyst Precursors in the One-Pot Four-Component Assembly of Oxo Triphenylhexanoates (OTHOs). Catalysts 2015, 5, 2052.

(54) Axelsson, A.; Ta, L.; Sundén, H. Direct Highly Regioselective Functionalization of Carbohydrates: A Three-Component Reaction Combining the Dissolving and Catalytic Efficiency of Ionic Liquids. Eur. J. Org. Chem. 2016, 2016 (20), 3339-3343.

(55) Hsu, C.-W.; Sauvée, C.; Sundén, H.; Andréasson, J. Writing and Erasing Multicolored Information in Diarylethene-Based Supramolecular Gels. Chem. Sci. 2018, 9 (41), 8019-8023.

(56) Johnstone, M. D.; Hsu, C.-W.; Hochbaum, N.; Andréasson, J.; Sundén, H. Multi-Color Emission with Orthogonal Input Triggers from a Diarylethene Pyrene-OTHO Organogelator Cocktail. Chem. Commun. 2020, 56 (6), 988-991.

(57) Tamai, S.; Ushirogochi, H.; Sano, S.; Nagao, Y. New Reaction Mode of the Dieckmann-Type Cyclization under the Lewis Acid-Et 3 N Conditions. Chem. Lett. 1995, 24, 295-296.

(58) Kamlar, M.; Runemark, A.; Císařová, I.; Sundén, H. Polycyclizations of Ketoesters: Synthesis of Complex Tricycles with up to Five Stereogenic Centers from Available Starting Materials. Org. Lett. 2020, 22 (21), 8387-8391.

(59) Evans, D. A.; Takacs, J. M.; Mcgee, L. R.; Ennis, M. D.; Mathre, D. J.; Bartroli, J. Chiral Enolate Design. Pure Appl. Chem. 1981, 53, $1109-1127$.

(60) Aggarwal, R.; Singh, G.; Sanz, D.; Claramunt, R. M.; Torralba, M. C.; Torres, M. R. NBS Mediated One-Pot Regioselective Synthesis of 2,3-Disubstituted Imidazo[1,2-a]Pyridines and Their Unambiguous Characterization through 2D NMR and X-Ray Crystallography. Tetrahedron 2016, 72 (27), 3832-3838.

(61) Ankner, T.; Fridén-Saxin, M.; Pemberton, N.; Seifert, T.; Grøtli, M.; Luthman, K.; Hilmersson, G. KHMDS Enhanced SmI2Mediated Reformatsky Type $\alpha$-Cyanation. Org. Lett. 2010, 12 (10), 2210-2213.

(62) Papageorgiou, C. D.; Ley, S. V.; Gaunt, M. J. Organic-CatalystMediated Cyclopropanation Reaction. Angew. Chem., Int. Ed. 2003, 42 (7), 828-831.

(63) Erhardt, H.; Mohr, F.; Kirsch, S. F. Synthesis of the 1,3,4Oxadiazole Core through Thermolysis of Geminal Diazides. Eur. J. Org. Chem. 2016, 2016 (34), 5629-5632. 
(64) El-Sayed, M. A.-A.; Abdel-Aziz, N. I.; Abdel-Aziz, A. A.-M.; ElAzab, A. S.; ElTahir, K. E. H. Synthesis, Biological Evaluation and Molecular Modeling Study of Pyrazole and Pyrazoline Derivatives as Selective COX-2 Inhibitors and Anti-Inflammatory Agents. Part 2. Bioorg. Med. Chem. 2012, 20 (10), 3306-3316.

(65) Molecular Operating Environment (MOE), ver. 2019.01; Chemical Computing Group ULC: Montreal, 2021. 